ARTICLE

https://doi.org/10.1038/s41467-018-08146-1

\title{
Partial proteasomal degradation of Lola triggers the male-to-female switch of a dimorphic courtship circuit
}

\author{
Kosei Sato (1) 1, Hiroki Ito², Atsushi Yokoyama ${ }^{3}$, Gakuta Toba ${ }^{4} \&$ Daisuke Yamamoto ${ }^{1,2}$
}

In Drosophila, some neurons develop sex-specific neurites that contribute to dimorphic circuits for sex-specific behavior. As opposed to the idea that the sexual dichotomy in transcriptional profiles produced by a sex-specific factor underlies such sex differences, we discovered that the sex-specific cleavage confers the activity as a sexual-fate inducer on the pleiotropic transcription factor Longitudinals lacking (Lola). Surprisingly, Fruitless, another transcription factor with a master regulator role for courtship circuitry formation, directly binds to Lola to protect its cleavage in males. We also show that Lola cleavage involves E3 ubiquitin ligase Cullin1 and 26S proteasome. Our work adds a new dimension to the study of sex-specific behavior and its circuit basis by unveiling a mechanistic link between proteolysis and the sexually dimorphic patterning of circuits. Our findings may also provide new insights into potential causes of the sex-biased incidence of some neuropsychiatric diseases and inspire novel therapeutic approaches to such disorders.

\footnotetext{
${ }^{1}$ Neuro-Network Evolution Project, Advanced ICT Research Institute, National Institute of Information and Communications Technology (NICT), 588-2 Iwaoka, Nishi-ku, Kobe 651-2492, Japan. ${ }^{2}$ Tohoku University Graduate School of Life Sciences, Sendai 980-8577, Japan. ${ }^{3}$ Tohoku University Graduate School of Medicine, Sendai 980-8575, Japan. ${ }^{4}$ Research Administration/Management Office, University of Tsukuba, Tsukuba 305-8577, Japan.

Correspondence and requests for materials should be addressed to K.S. (email: kosei@nict.go.jp) or to D.Y. (email: daichan@nict.go.jp)
} 
F emales and males display distinct behavioral patterns. These differences in behavioral patterns are the outcome of the intersex differences in neural circuits ${ }^{1}$. In Drosophila, it has well been documented that sexual dimorphisms in neural circuits result, in principle, from sex differences in the number, structure and function of individual cells composing the circuit $^{2-6}$. The development of these cellular sex differences relies primarily on either or both of two sex determination genes, fruitless (fru) and doublesex $(d s x)^{5}$. However, it is largely unknown how fru and $d s x$ produce sex differences in individual cells. We discovered that the longitudinals lacking (lola) gene is an essential mediator for the fru action to masculinize the mAL cell structure. The lola locus generates extraordinarily large numbers of mRNA variants (Supplementary Fig. 1), each of which encodes a unique protein isoform. The majority of Lola isoforms share an N-terminal BTB domain implicated in protein-protein interactions ${ }^{7}$ and in anchoring ubiquitin proteasome components, followed by an isoform-specific sequence containing a C-terminal zinc finger motif that is a putative DNA-binding region ${ }^{8}$. Interestingly, the fru gene product FruM represents another, BTB-zinc finger protein group, which includes a set of male-specific proteins (i.e., FruAM, FruBM and FruEM: nomenclature according to Ref. 9; Supplementary Fig. 1) that function to masculinize certain neurons ${ }^{10-12}$ presumably via chromatin remodeling 9 . For example, FruM represses transcription from roundabout1 (robo1), a negative regulator gene for neuritogenesis, thereby allowing malespecific neurite formation in males ${ }^{13}$. Here we demonstrate that the male-biased Lola29M isoform forms a complex with FruBM. We further demonstrate that Lola29M is a precursor of the female-specific Lola29F isoform, an N-terminal truncation product of Lola29M that counteracts Lola29M action so as to inhibit male-specific neurite formation in females. Surprisingly, the male-specific transcriptional repressor FruBM protects Lola29M from its N-terminal truncation, which is mediated by the E3 ubiquitin ligase Cullin1 (Cul1) and 26S proteasome. Thus, by making the masculinizing protein Lola $29 \mathrm{M}$ resistant to degradation, the male-specific transcription factor FruBM prevents the production of the feminizing protein Lola29F. As a consequence, the male program for dimorphic circuit formation turns on and the female program turns off in the male brain.

In humans, sexually biased incidence of certain neurological disorders has been well recognized, yet the origin of such sex differences remains largely an enigma. For instance, the male-tofemale incidence ratios have been reported to vary from 1.37 to 3.7 in Parkinson's disease, the etiology of which likely involves mitochondrial dysfunction due to defects in the proteasomal degradation system ${ }^{14}$. Our finding in Drosophila that the neuronal sex-type specification involves proteasomal protein processing will shed light on the hitherto unknown mechanistic link among posttranslational protein modification, neural sex differentiation and complex neurobehavioral traits under normal and disordered conditions.

\section{Results}

lola as a phenotypic modifier of $f r u$. To obtain insights into the mechanism of fru actions on neural sex-type specification, we here screened for fru modifier genes. In this screen, we took advantage of a gain-of-function effect of $\mathrm{fru}^{+}$to disrupt the compound eye structure when overexpressed in the developing eye disc. Genome-wide searches for genes that can modify the fru eye phenotype were conducted by the Gene-Search (GS) system $^{15}$. In this system, a P-element vector carrying the GAL4responsive DNA sequence UAS (the GS-element) was randomly inserted into the genome of a fly so that transcription units that happened to flank the GS-element insertion could be transcribed in the presence of an arbitrarily chosen GAL4 transgene (Supplementary Fig. 2a). This study used GMR-GAL4 to drive transcription via the GS-element as well as $U A S-f r u B^{+}$in the developing eye disc (Supplementary Fig. 2b), yielding several enhancers of the $f r u B^{+}$-induced eye phenotype, which included lola (Supplementary Fig. 2c-e; for other fru modifiers see Ref. 16). Conversely, a loss-of-function lola mutation dominantly suppressed the fru-induced distortion of the eye (Supplementary Fig. $2 \mathrm{f}, \mathrm{g})$. In subsequent analyses, we will focus on lola because: (1) it encodes proteins of the BTB-zinc finger superfamily to which Fru also belongs and (2) its functions in neurite guidance have been well established ${ }^{17}$. Indeed, we found that reduced courtship toward a female in fru hypomorphic males was dominantly enhanced by two different lola null alleles (lola ${ }^{03089}$ and lola ${ }^{\mathrm{ORE}} 76$ ), which are both homozygous lethal ${ }^{18,19}$ (Fig. 1a), and lola knockdown per se attenuated male courtship activities (Fig. 1b). These results implicate lola in the fru-dependent formation of courtship circuits.

Lola isoforms dedicated to sexual differentiation. We suspected that Fru (more specifically, FruBM, see below) might affect expression of lola to exert its neural masculinizing effect in, for example, sexually dimorphic mAL interneurons in the brain (Supplementary Fig. 2h), although no sex differences in the structure or expression of Lola isoforms has been reported and despite the numerousness of Lola isoforms identified to date ${ }^{20}$. To obtain hints as to which of the Lola isoforms might have a role in the fru-dependent sexual differentiation, we examined possible effects of isoform-specific knockdown for isoforms 11, 17, 22, 26, 28 and 29 , for which UAS-RNAi transgenic strains were publicly available, and found that isoforms 22 and 29 interfered with the sex-specific development of fru-expressing neurons (see below). Our extensive analyses of Lola expression with an antibody raised against an exon29-specific sequence (anti-Lola-exon 29; Supplementary Fig. 3) revealed two isoforms, Lola29M of $\sim 110 \mathrm{kD}$ and Lola $29 \mathrm{~F}$ of $\sim 80 \mathrm{kD}$, the relative amounts of which were different between the sexes in wandering stage third instar larvae (Fig. 1c). The isoform containing the sequence encoded by exon 29 was formally designated as type- $\mathrm{Q}^{20}$. We found that Lola29M is more abundant in males than females whereas Lola29F is detectable only in females (Fig. 1d). Immunostaining of the wandering-stage larval CNS with the anti-Lola-exon 29 antibody revealed that Lola29M/F expression is confined to differentiating neurons, including those expressing FruM, but not detected in neuroblasts (Supplementary Fig. 4). The Lola29M and Lola29F isoforms shared exon29, which was connected to the same 5' exons, and transcribed from the same promoters. Because no sex difference was detected in the mRNA species with exon 29 by $5^{\prime}$ and $3^{\prime}$ RACE (Supplementary Fig. 5), the size difference between the Lola29F and Lola29M proteins was considered to result from posttranslational modification of a protein product. Intriguingly, fru mutant $\left(\right.$ rru $\left.^{-}\right)$males that are null for male-specific FruM proteins expressed the female-specific Lola29F isoform with a concomitant reduction in Lola29M (Fig. 1c, d). In contrast, females sexually transformed into males by the transfor$\mathrm{mer}^{1}\left(\mathrm{tra}^{1}\right)$ mutation lost Lola29F with an elevated level of Lola29M (Fig. 1c, d). These results imply that the posttranslational modification of the Lola protein is under the control of the sex-determination cascade downstream of tra and fru.

Female-specific Lola29F is a truncation product of Lola29M. To examine whether female-specific Lola29F is a truncation product of male-enriched Lola $29 \mathrm{M}$, we attempted to determine the structures of Lola29F and Lola29M and the mechanism whereby these two isoforms are produced in a sex-dependent 
a

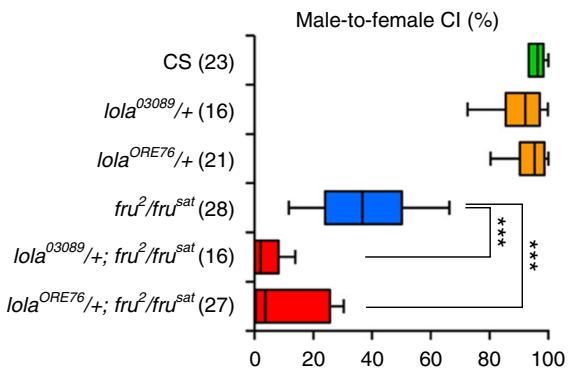

b

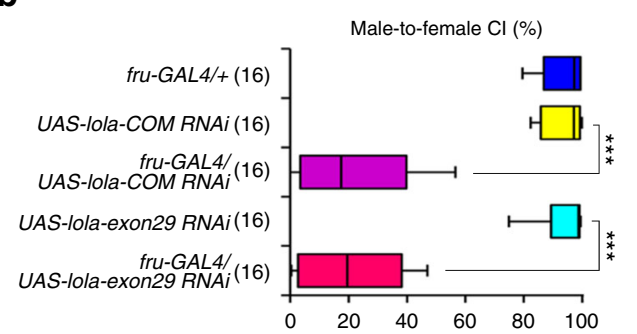

C

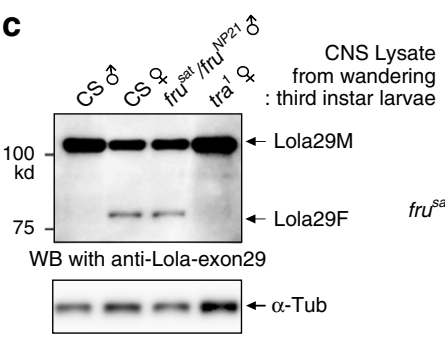

d

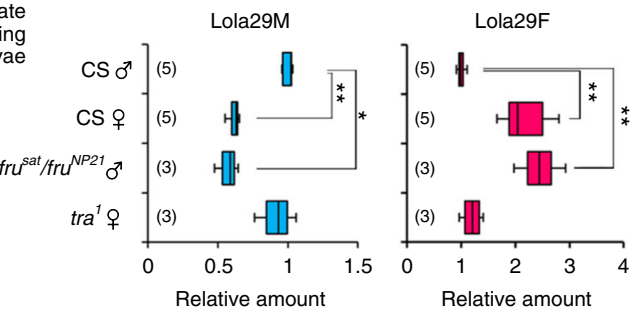

e

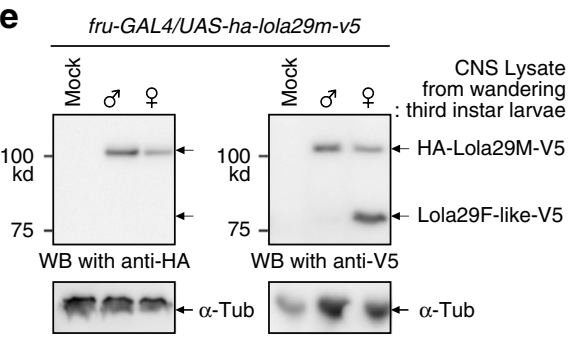

f $f$ ru-GAL4/UAS-Iola29m[41-300]-v5

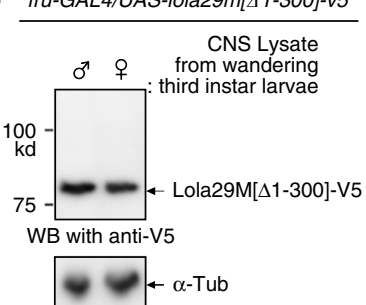

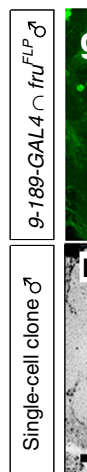
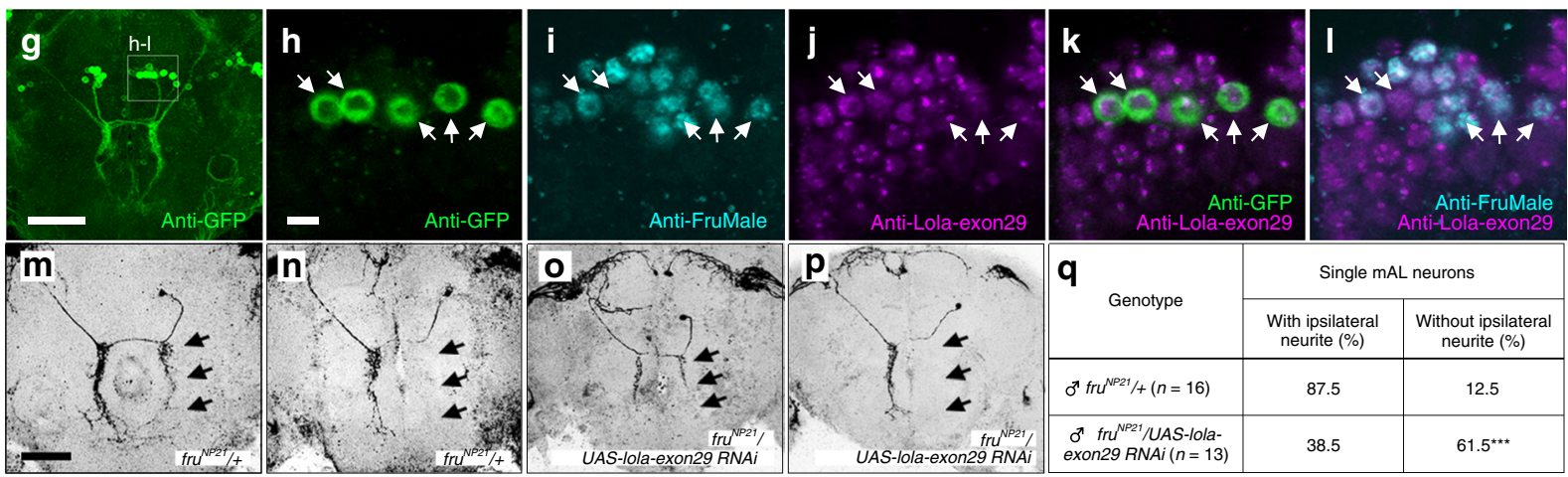

Fig. 1 lola exon 29 contributes to a set of Lola isoforms differentially expressed between females and males. a, b Male courtship activities. Courtship defects in fru hypomorphic males were enhanced in the lola heterozygous background (a) and knockdown by lola-COM RNAi (3rd bar from the top) or by lola-exon 29 RNAi (5th bar from the top) suppressed male-to-female courtship (b). ${ }^{\star \star \star} P<0.001$ by the Kruskal-Wallis analysis of variance followed by Steel-Dwass

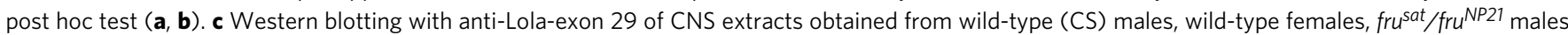
and tra ${ }^{1}$ homozygous females. d Quantification of Lola29M (left-side panel) and Lola29F (right-side panel), as normalized by the values for the wild-type male. The number of replicates is indicated in parentheses. ${ }^{\star \star} P<0.01 ;{ }^{\star} P<0.05$ by the Kruskal-Wallis analysis of variance followed by Steel's nonparametric multiple comparisons. The box plot shows the median and 10th, 25th, 75th, and 90th percentiles. e Overexpression of a Lola29M-encoding sequence decorated with a HA-tag at the N-terminus and a V5-tag at the C-terminus (HA-lola29m-V5) yielded the Lola29M protein in males and less abundantly in females, as well as a Lola29F-like protein in females. Shown is a western blot of larval CNS extracts probed with anti-V5 that recognizes the C-terminus of Lola29M. f Lola29M[ 11-300] lacking a.a. 1-300 resulted in a protein, Lola29F-like, with a molecular weight close to that of Lola29F in both sexes. $\alpha$-Tubulin ( $\alpha$-Tub) was used as a loading control. $\mathbf{g}$-I mAL neurons in the male brain triply stained with antibodies against GFP (g, $\mathbf{h}, \mathbf{k}$ ), FruM (i, I) and Lola29M/F (j-I). GFP was preferentially expressed in mAL neurons by the intersection of fruFLP and 9-189-GAL4. Scale bars: $50 \mu \mathrm{m}(\mathbf{g})$ or $10 \mu \mathrm{m}(\mathbf{h})$. $\mathbf{m}-\mathbf{q}$ Analysis of single-cell mAL clones that express lola-exon29 RNAi. Examples of single-cell clones (m-p) and the proportion of flies that carried clones with or without the ipsilateral neurite $(\mathbf{q})$. Scale bar: $50 \mu \mathrm{m} .{ }^{\star \star \star} P<0.001$ by the Fisher's exact probability test. Source data are provided as a Source Data file

manner. We presumed that Lola29F might be a proteolytic product of Lola29M. To test this idea, we overexpressed a lola29m gene decorated with an N-terminal HA tag and a C-terminal V5 tag in flies under the control of fru-GAL4, and analyzed the lysates from CNS cells by western blotting with antibodies against HA and V5 (Fig. 1e). Remarkably, the anti-V5 antibody detected a shorter band similar to Lola29F (referred to as Lola29F-like hereafter) in addition to the full-length Lola29M, whereas the anti-HA antibody detected only the latter (Fig. 1e). Therefore, the difference between the two isoforms must reside in their $\mathrm{N}$ termini. Overexpression of a series of N-terminal deletants of lola29m in fru-GAL4-positive cells resulted in the expression of mutant Lola29 proteins; deletion of the N-terminal $300(\Delta 1-300)$ residues yielded a single band of a size similar to that of Lola29F on western blots (Fig. 1f). We infer that Lola29F is produced by the truncation of Lola29M somewhere between residues 250 and 300 of Lola29M, and later analyses by Edman degradation revealed that this is indeed the case (see below).

Because lola plays an important role in neurite patterning, the effects of lola knockdown on courtship behavior (Fig. 1b) likely result from a disturbance in the formation of courtship circuits. It has been established that FruM plays a key role in the courtship circuit formation in the male CNS, and some of the fru mutant defects in mating behavior have been ascribed to impairments of sex-specific development of fru-expressing neurons ${ }^{10-12}$. Therefore, to examine the possible role of Lola29F/M on the courtship circuit formation, we next examined fru-expressing $\mathrm{mAL}$ interneurons, which exhibit remarkable sex-differences 
(Supplementary Fig. 2h), which have been shown to play a role in the processing of sex pheromone information ${ }^{21-23}$. One of the prominent sex differences found in mAL neurons is the absence (in females) or presence (in males) of the ipsilateral neurite. In a subsequent analysis, therefore, we focused on the male-specific ipsilateral neurite formation, where male-specific FruM directly represses transcription of robo1 in males, thereby allowing $\mathrm{mAL}$ neurons to extend the male-specific neurite ${ }^{13}$. Gain of the malespecific ipsilateral neurite (i.e., masculinization) in females or fruM-null mutant males can be assessed in neuroblast clones that contain up to $5 \mathrm{mAL}$ neurons, because none of the mAL neurons in control females and control fruM-null males possesses this neurite. Loss of the male-specific neurite (i.e., feminization) in males, in contrast, is only detectable in single-cell clones, because any remaining ipsilateral neurite of unaffected cells hinder the neurite loss from affected cells ${ }^{13}$. We therefore examine neuroblast clones for quantifying the masculinizing effect of genetic manipulations and single-cell clones for the feminizing effect.

We first confirmed the immunoreactivity of mAL neurons to the anti-Lola-exon 29 antibody (Fig. 1g-l). We then tested the effect of lola29 knockdown in these cells. Notably, the expression of RNAi targeting lola-exon 29 markedly increased the formation of $\mathrm{mAL}$ neurons without the male-specific ipsilateral neurite in male mAL neurons (Fig. $1 \mathrm{~m}-\mathrm{q})$. It is noteworthy that the lolaexon29 RNAi used here effectively inhibited Lola29M production in flies, which retained a high level of expression of other lola isoforms (Supplementary Fig. 6).

To further clarify roles of Lola29M and Lola29F in the sex-type specification of neurites, we first wanted to define their molecular structures and the mechanism whereby two Lola29 forms are produced. To determine the exact site of truncation in producing Lola29F from Lola29M, we employed Edman degradation analysis (Fig. 2a). To obtain a large amount of Lola29F sufficient for amino acid sequencing, we overexpressed lola29m-6xV5 under the control of fru-GAL4 in female larvae, from which the CNSs were dissected to extract proteins for immunoprecipitating lola $29 m-6 x V 5$ products. The band representing Lola $29 \mathrm{~F}$ on the PVDF membrane visualized with Coomassie brilliant blue (CBB; Fig. 2a) was cut out and analyzed, allowing us to determine that the N-terminal five residues were SSTAA (Fig. 2b). This fiveresidue sequence was uniquely identified as a.a. 264-268 in Lola29M. We therefore conclude that a.a. 1-263 of Lola29M are degraded to yield Lola29F.

In a separate experiment, we detected Lola29F-like, in addition to the expected Lola29M, in S2 cells that had been transfected with lola29m alone without a lola29f minigene when lysates were prepared with a sufficient delay ( 2 days) after transfection (Supplementary Fig. 7). Importantly, the production of Lola29Flike in S2 cells was inhibited by the administration of a ubiquitinproteasome inhibitor, MG-132 (Fig. 2c) or Lactacystin (Fig. 2d). This result suggests that Lola29F-like is produced by ubiquitinmediated proteolysis of Lola29M in S2 cells.

Polyubiquitination of ubiquitin at lysine 48 (K48) is known to direct substrate proteins to proteasome-mediated degradation ${ }^{24}$. We found that a K48-linkage-specific polyubiquitin antibody coimmunoprecipitates Lola29M/F in lysates from S2 cells transfected with lola29m, and fruBM cotransfection markedly diminishes the yield of immunoprecipitates (Supplementary Fig. 8). This result is taken as evidence that Lola29M is indeed ubiquitinated in the absence of FruM.

Ubiquitination takes place at lysine $(\mathrm{K})$ residues. Lola29M has 40 lysine residues, and 15 of them are within the N-terminal 263 amino acid region, which is likely removed in Lola29F (Fig. 2e). We replaced these 15 lysine residues with arginine (R) one by one to examine whether any of these mutations confer to Lola29M the resistance against proteolysis. Notably, four mutants, K41R, $\mathrm{K} 44 \mathrm{R}, \mathrm{K} 47 \mathrm{R}$ and $\mathrm{K} 67 \mathrm{R}$, showed a marked reduction in the Lola29F-like production (Fig. 2f). The K41R mutation similarly protected Lola29M from proteolysis in vivo (Fig. $2 \mathrm{~g}$ ). Taking all these results into account, we conclude that Lola29F is a proteolytic product of Lola29M (Fig. 2h).

Lola29M masculinizes whereas Lola29F feminizes the neurons. Based on knowledge of their molecular properties, we attempted to determine the roles of Lola29M and Lola29F on sex-specific neurite identity. We found that overexpression of Lola29F-like inhibits the ipsilateral neurite formation; the proportion of singlecell clones without the ipsilateral neurite was significantly increased upon Lola29F-like overexpression in males (Fig. 3a-c). However, mAL neurons with the ipsilateral neurite were still produced, though at a reduced rate, in male flies with Lola29Flike overexpression, implying that an additional, Lola29Fresistant mechanism operates for the ipsilateral neurite formation.

We next examined the effect of overexpression of Lola29M (truncation-resistant Lola29M[K41R]) and Lola29F-like (Nterminally truncated Lola29M[ $\Delta 1-300]$ ) in females (Supplementary Fig. 9) and fruM-null mutant males (Fig. 3d-h), which lack endogenous FruM that could potentially complicate the analysis. Overexpression of Lola29M induced the male-specific ipsilateral neurite in some females (Supplementary Fig. 9b) and some fru mutant males (Fig. 3e), which otherwise lack it without exception (Fig. 3d and Supplementary Fig. 9a). It is conceivable that endogenous Lola29F present in females and fru mutant males (see Fig. 1c) hampered the masculinizing action of overexpressed Lola29M[K41R], such that formation of the ipsilateral neurite in mAL neurons was observed in only $20-40 \%$ of flies examined (Fig. 3h and Supplementary Fig. 9d). This notion was supported by the observation that the ability of Lola29M[K41R] to restore the male-specific neurite in fru mutant males was canceled out by the additional overexpression of Lola29F-like (Fig. 3g). In contrast, overexpression of Lola29F-like alone via an $\mathrm{N}$ terminally deleted lola29m[ $\Delta 1-300]$ transgene in females (Supplementary Fig. 9c) or fru mutant males (Fig. 3f) had no effect on the mAL neurite structure, which continued to be the female-type in all flies examined.

To examine whether Lola29M and Lola29F are involved in the neural sexual differentiation of neurons other than $\mathrm{mAL}$, we overexpressed truncation-resistant Lola29M or Lola29F-like in fru-expressing pheromone receptor neurons (Fig. $4 \mathrm{a}-\mathrm{g}$ ), the central projection of which is sexually dimorphic; male afferent fibers cross the midline (Fig. $4 \mathrm{a}, \mathrm{b}$ ) whereas female counterparts terminate in the ipsilateral neuromere in the prothoracic ganglion $^{25}$. fru hypomorphic mutant $\left(f r u^{2} / f r u^{s a t}\right)$ males have a reduced midline crossing (Fig. 4c), offering a sensitized genetic condition in which subtle phenotypic changes can be unambiguously detected $^{9}$. We found that truncation-resistant Lola29M significantly increased (Fig. 4d) and Lola29F-like significantly decreased (Fig. 4e) the midline crossing of sensory axons (Fig. 4g), suggesting that Lola29M and Lola29F contribute to certain dimorphic features of different types of fru-expressing neurons. We conclude that Lola29M promotes the male-typical neurite formation and Lola29F counteracts the action of Lola29M.

Lola29F and Lola29M are required for mating behavior. To evaluate the contributions of Lola29M and Lola29F to the execution of mating behavior, we measured the courtship index for male courtship vigor and the time to copulation for female receptivity in flies which expressed lola exon 29-RNAi together with lola $29 m[K 41 R]$ encoding truncation-resistant Lola29M or 
a

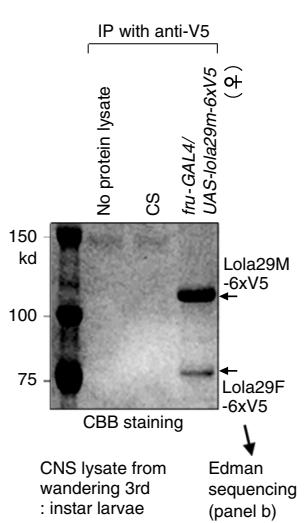

C

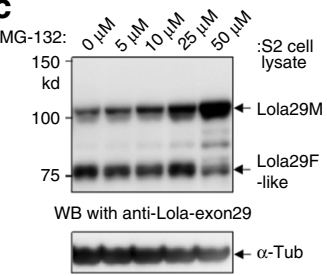

d

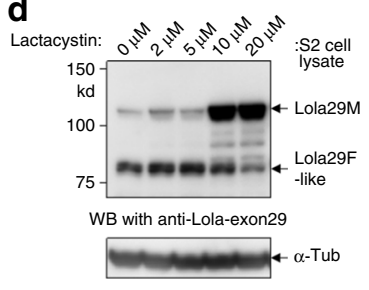

e

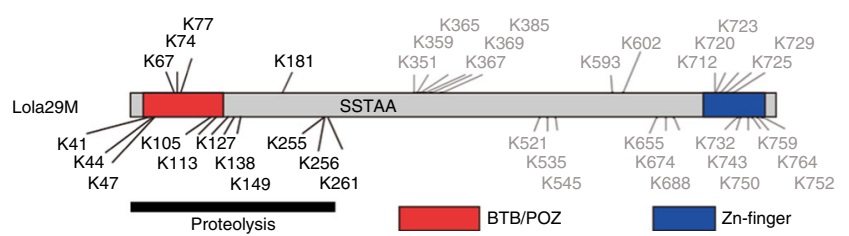

b
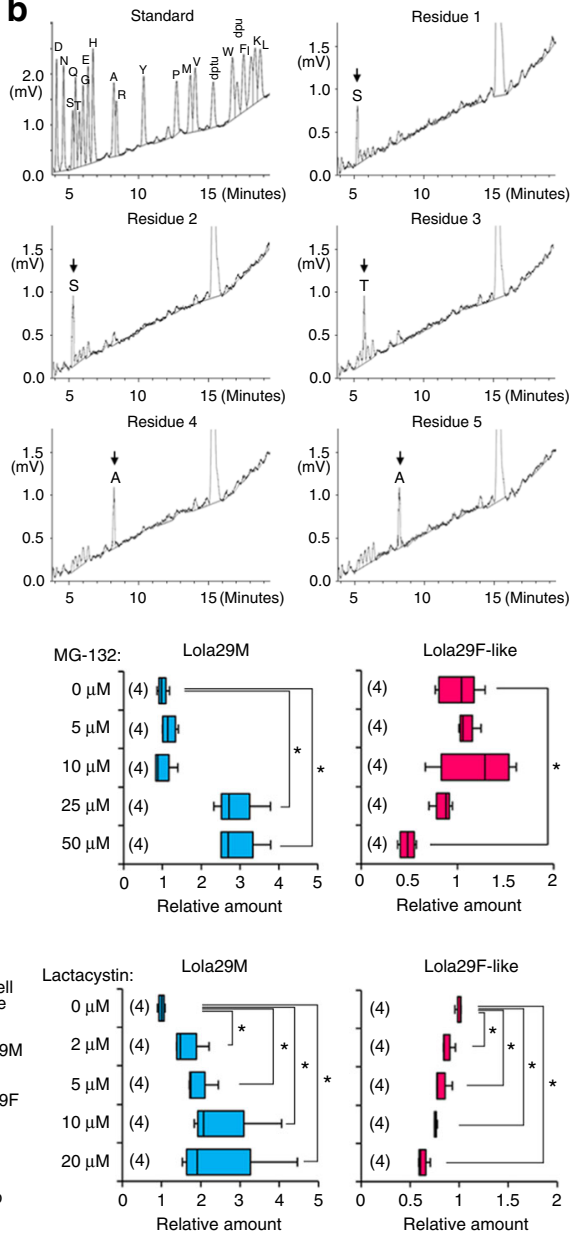

Zn-finger

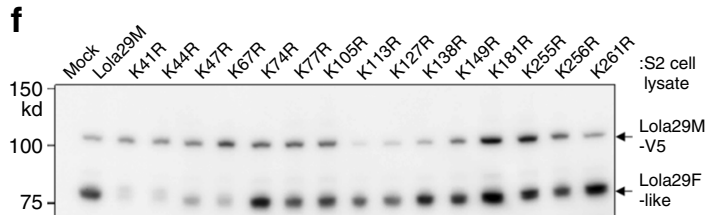

WB with anti-V5

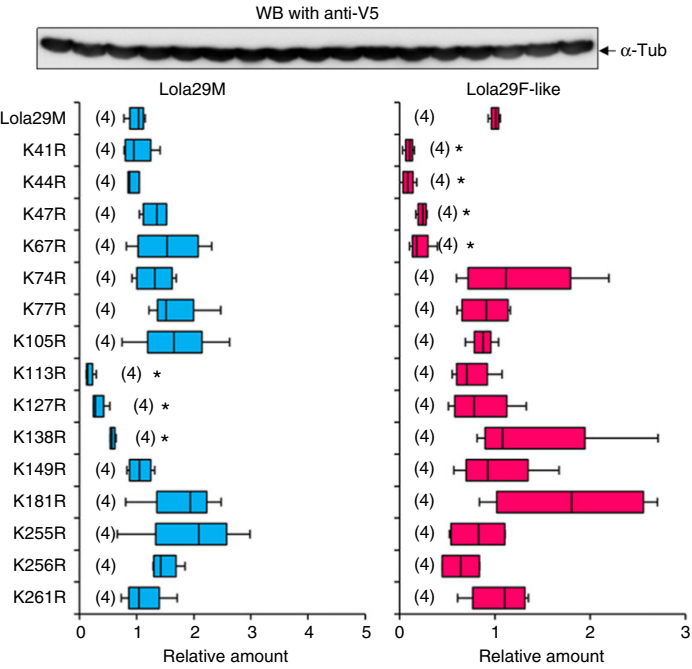

g
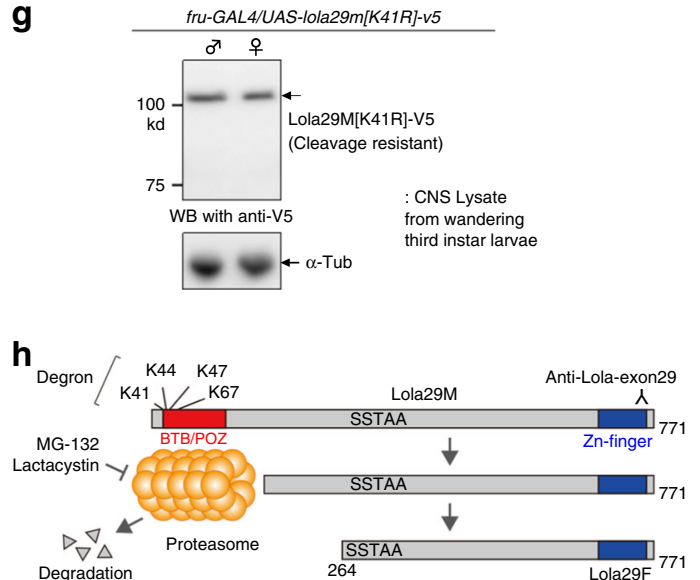

Fig. 2 Proteasome involvement in the production of Lola29F. a A coomasie brilliant blue (CBB) stained PVDF membrane showing results from the biochemical purification of Lola29M and Lola29F. Iola29m-6xv5 was overexpressed via fru-GAL4 in female larvae, from which the CNSs were dissected to extract proteins for immunoprecipitation. The band representing Lola29F (arrow) was transferred to a PVDF membrane and subjected to Edman sequencing. b HPLC profiles of Edman sequencing of Lola29F. The 20 known amino acids served as standard (top-left panel). The first five amino acids of the N-terminal region of Lola29F were determined to be SSTAA, which corresponds to the a.a.264-a.a.268 in Lola29M. c, d Western blotting of S2 cell extracts probed with anti-Lola-exon 29 (upper panel). Administration of proteasome inhibitors MG-132 (c) or lactacystin (d) reduced the amount of Lola29F-like. $\alpha$-Tubulin was used as a loading control (lower panel). Effects of inhibitors on the amounts of Lola29M and Lola29F-like are shown on the right-hand side. ${ }^{\star} P<0.05$ by the Kruskal-Wallis analysis of variance followed by Steel's nonparametric multiple comparisons. e Distribution of lysine residues throughout the Lola29M protein. $\mathbf{f}$ Western blot analysis of transfected S2 lysates for Lola29M and Lola29F-like revealed a marked decline in the amount of Lola29F-like with K41R, K44R, K47R and K67R substitutions. ${ }^{\star} P<0.05$ by the Kruskal-Wallis analysis of variance followed by Steel's nonparametric multiple comparisons. $\mathbf{g}$ Shown is a western blot of extracts obtained from the larval CNS probed with anti-V5 that recognizes the Cterminus of a Lola29M variant. Replacement of the lysine residue at a.a. 41 with arginine (K41R) in the Lola29M-encoding sequence blocked the production of Lola29F in females (right lane). $\mathbf{h}$ The hypothesis that Lola29F is derived from Lola29M by truncation of the N-terminus with a BTB domain. Edman degradation analysis identified the N-terminus of Lola29F-like as SSTAA, which corresponds to a.a. 264-268 of Lola29M. The four distal-most lysine residues $(K 41, K 44, K 47$, and $\mathrm{K} 67)$ are postulated to form a degron, a set of degradation signals. The box plot shows median and 10 th, 25 th, 75 th, and 90 th percentiles $(\mathbf{c}, \mathbf{d}, \mathbf{f})$. Source data are provided as a Source Data file

lola29m[A1-300] encoding Lola29F-like in fru-GAL4-positive cells. We found that lola exon 29-RNAi effectively reduced the courtship index to $\sim 40 \%$ of the value in control males (Fig. $4 \mathrm{~h}$ ), a level of inhibition comparable to that obtained with lola-COM $R N A i$, which knocked down nearly all types of lola mRNAs
(Fig. 1b). As mentioned above, our western blot analysis confirmed that both lola-COM RNAi and lola-exon29 RNAi effectively reduced Lola29M expression (Supplementary Fig. 6). Importantly, Lola29M[K41R] significantly ameliorated the suppressive effect of lola-exon 29 RNAi on male courtship activities, 


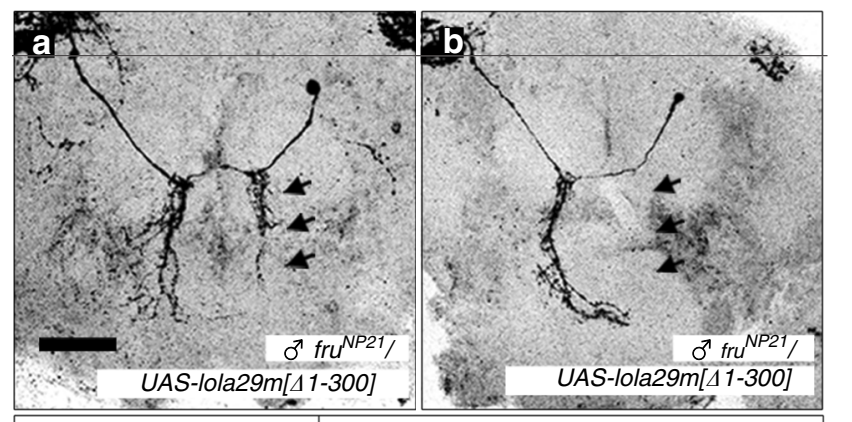

\begin{tabular}{|c|c|c|}
\hline \multirow{2}{*}{ Genotype } & \multicolumn{2}{|c|}{ Single $\mathrm{mAL}$ neurons } \\
\hline & $\begin{array}{c}\text { With ipsilateral } \\
\text { neurite (\%) }\end{array}$ & $\begin{array}{l}\text { Without ipsilateral } \\
\text { neurite (\%) }\end{array}$ \\
\hline$\sigma^{7} f r u^{N P 21} /+(n=16)$ & 87.5 & 12.5 \\
\hline $\begin{array}{l}\sigma^{7} \mathrm{fru}^{N P 21} / \mathrm{UAS}- \\
\text { lola29m[ } \\
(n=12)\end{array}$ & 50.0 & $50.0^{*}$ \\
\hline
\end{tabular}
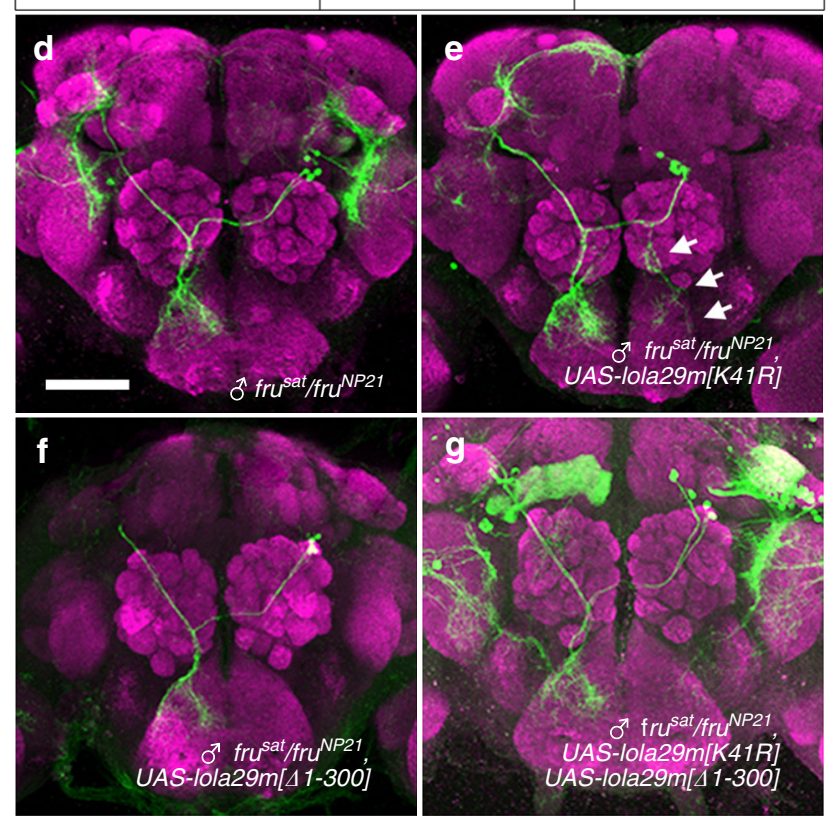

h

$\%$ of mAL clones carrying ipsilateral neurites

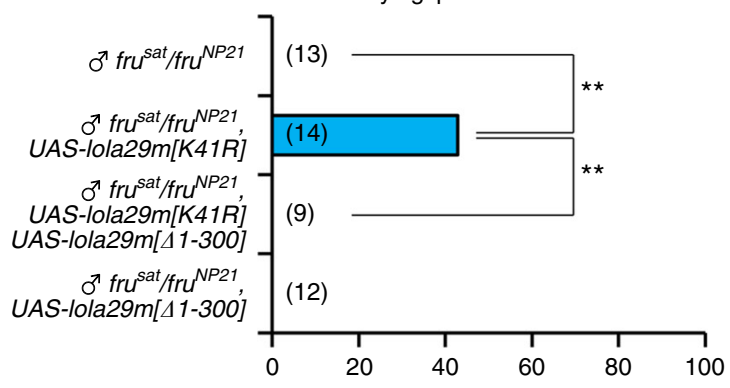

whereas Lola29F-like failed to do so (Fig. 4h). Conversely, in females, Lola29F-like overexpression via fru-GAL4 mitigated the inhibitory effect of lola-exon 29 RNAi expression on female mating, whereas Lola29M[K41R] did not (Fig. 4i, j). Because male
Fig. 3 Lola29M promotes whereas Lola29F impedes male-typical neuritogenesis. a-c Analysis of single-cell $\mathrm{mAL}$ clones overexpressing Lola29F-like. Examples of single-cell clones $(\mathbf{a}, \mathbf{b})$ and the proportion of flies that carry single-cell $\mathrm{mAL}$ clones with or without the ipsilateral neurite (c). Scale bar: $50 \mu \mathrm{m}$. Statistical differences were evaluated by the Fisher's exact probability test $\left({ }^{\star} P<0.05\right)$. $\mathbf{d}-\mathbf{h} \mathrm{mAL}$ neurons in fru mutant (FruM protein-null) males without (d) or with overexpression of truncationresistant Lola29M[K41R] (e) or Lola29F-like (f), or both (g). Representative MARCM clones are shown. $\mathbf{h}$ Quantitative comparisons of the percentage of $\mathrm{mAL}$ neuroblast clones that carried ipsilateral neurites ${ }^{\star \star} P<0.01$ by the Fisher's exact probability test. Scale bars: $50 \mu \mathrm{m}$

flies paired with test females of different genotypes were equally active in courtship (Fig. 4k), the observed reduction in mating success in this experiment was ascribable to a change in female receptivity. These results suggest that Lola29F and Lola29M play sex-specific functions in females and males, respectively, to ensure successful courtship and copulation. We note, however, that neither Lola29M nor Lola29F-like was able to resume the mating activity to the normal level in male or female flies expressing lolaexon29 RNAi. The partial rescue of mating behavior by overexpressed Lola29M or Lola29F-like might suggest that mRNAs derived from the transgenes were also targeted by the lola-exon 29 $R N A i$ to a certain extent.

Lola29F prevents Lola29M from binding to its target. To explore the mechanism by which Lola29F counteracts Lola29M, we first examined the possible effect of Lola29F/M on the transcription of robo1, a direct downstream target gene of FruBM ${ }^{13}$. Of note, Crowner et al. ${ }^{18}$ have reported that a robol mutant copy dominantly enhances the axon misrouting phenotype in a weak hypomorph of lola, lola ORE120, which barely manifests this phenotype on its own. Our quantitative RT-PCR experiment with male fly extracts revealed that overexpression of Lola29M decreased robo1 transcripts, whereas overexpression of Lola29Flike had no effect on its own (Fig. 4l). Interestingly, the reduction in robo1 transcript levels by Lola29M was not detected when Lola29F-like was co-overexpressed (Fig. 4l). In keeping with the RT-PCR result, overexpression of Lola29M[K41R] in the larval CNS reduced its immunoreactivity to the anti-Robol antibody (Supplementary Fig. 10). These results support the hypothesis that Lola29F inhibits the transcriptional repressor activity of Lola29M.

To further explore the mechanism by which Lola $29 \mathrm{~F}$ counteracts Lola29M, we conducted reporter assays. A series of reporters with a luciferase-coding sequence fused to robol promoter fragments of varying length (Fig. 5a) were transfected into S2 cells with or without Lola29M and/or FruBM. The reporter activity was repressed by Lola29M, irrespective of whether FruBM was present (Fig. 5b), provided that the reporter contained a robol promoter fragment of $0.9 \mathrm{~kb}$ or longer (Fig. 5c). Truncation-resistant Lola29M[K41R] was equally effective as wild-type Lola29M in repressing the robo1 reporter activity (Fig. 5c). Notably, Lola29F-like encoded by lola29m[ $\Delta 1-300]$ had no effect on the robo1 reporter activity (Fig. 5d). Intriguingly, the ability of Lola29M to repress reporter activities was completely blocked by the addition of Lola29F-like (Fig. 5e).

An in silico search for the putative Lola-binding motif within the Lola-responsive $0.9 \mathrm{~kb}$ region of the robol promoter revealed an 18-bp direct repeat (DR), G C A C T A A A G A G C A G G A A A, which we named DR1 (Fig. 5f, g). The $0.9 \mathrm{~kb}$ reporter construct lost its sensitivity to Lola29M when DR1 was deleted from the promoter fragment (i.e., the $0.9 \mathrm{~kb} \Delta \mathrm{DR} 1$ reporter; Fig. 5h). Interestingly, DR1 was located immediately 3' to Pal 1, a palindrome sequence shown to be essential for a 42-bp 

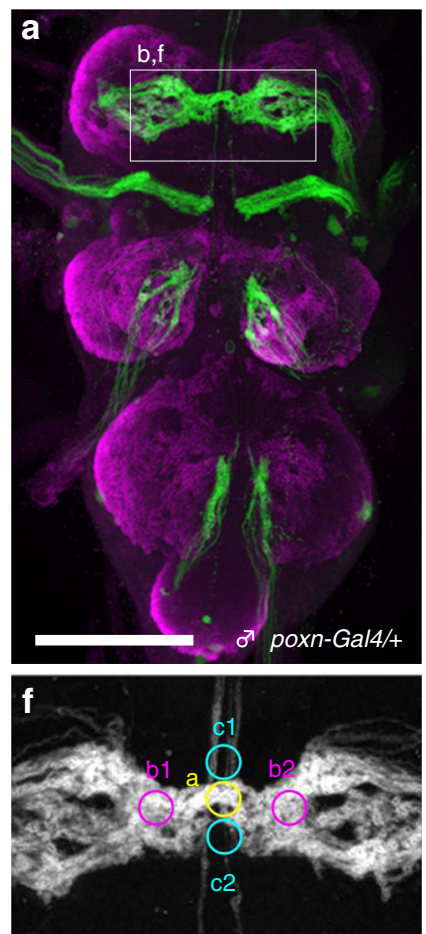

$\underset{\text { score }}{\operatorname{Midline~crossing~}}=\frac{\mathrm{a}-(\mathrm{c} 1+\mathrm{c} 2) / 2}{(\mathrm{~b} 1+\mathrm{b} 2) / 2}$

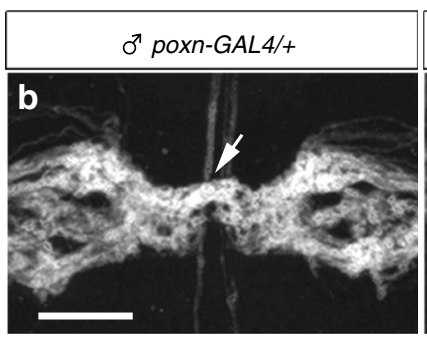

$\sigma^{7} \mathrm{fru}^{2}$, UAS-lola29m[11-300]
/fru sat poxn-GAL4

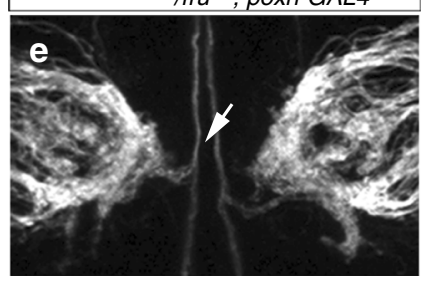

h

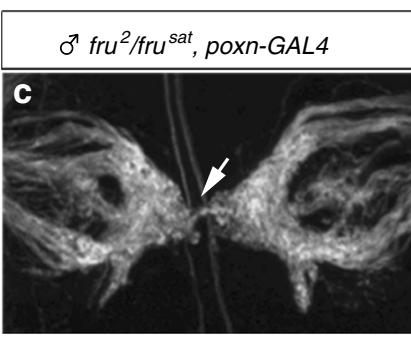

g

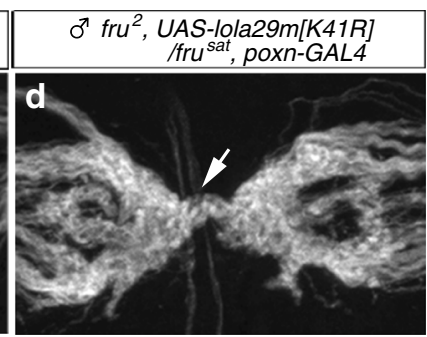

Midline crossing score

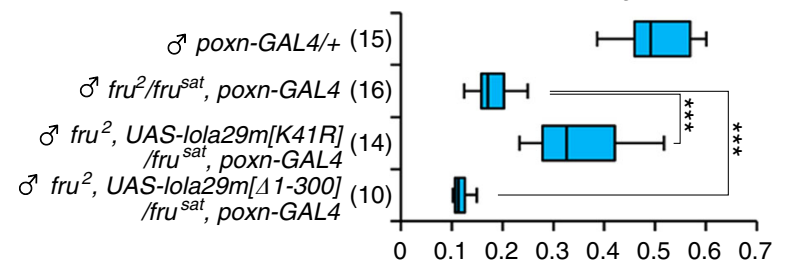

Male-to-female $\mathrm{Cl}(\%)$

fru-GAL4/+ $\sigma^{7}(16)$

UAS-Iola-exon29 RNAi ठৃ (16)

fru-GAL4/UAS-Iola-exon29 RNAi ठ (16)

fru-GAL4/UAS-Iola-exon29 RNAi, UAS-lola29m ơ (16)

fru-GAL4/UAS-Iola-exon29 RNAi, UAS-Iola29m[K41R] ठౌ (16)

fru-GAL4/UAS-Iola-exon29 RNAi, UAS-Iola29m[41-300] ठ (16)

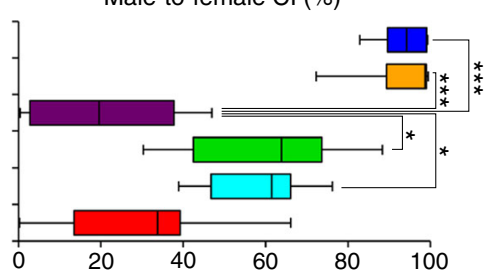

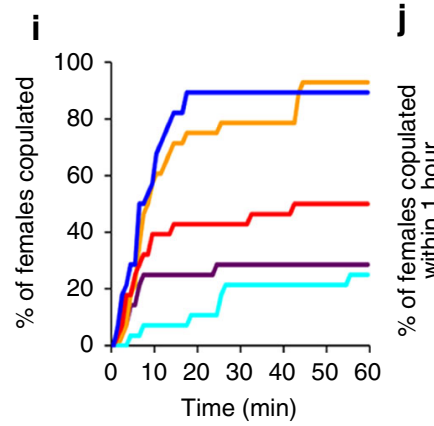

- fru-GAL4/+ 우

- UAS-lola-exon29 RNAi우

- fru-GAL4/UAS-Iola-exon29 RNAi 우

- fru-GAL4/UAS-Iola-exon29 RNAi, UAS-lola29m[K41R] 우

- fru-GAL4/UAS-Iola-exon29 RNAi, UAS-Iola29m[41-300] 우

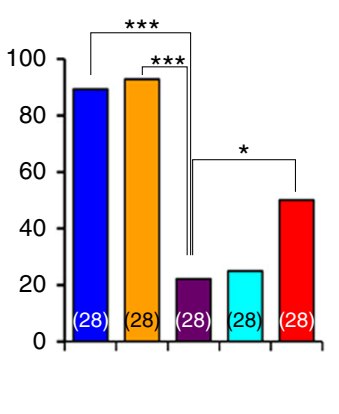

k

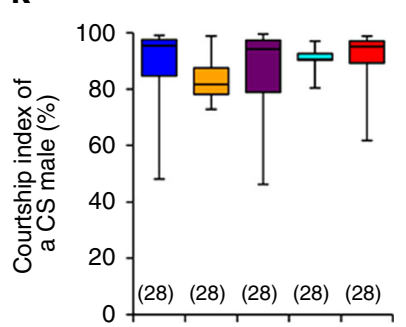

I

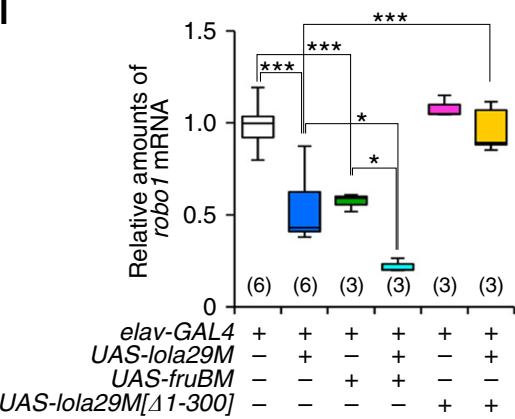

Fig. 4 Lols29F/M are required for mating behavior. a-g Effect of Lola29M or Lola29F-like overexpression on midline crossing of leg sensory afferents. The

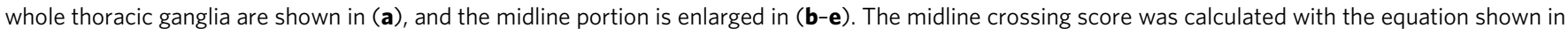
(f) with values of fluorescent intensity measured at five regions, a, b1, b2, c1 and c2, near the midline. $\mathbf{g}$ Comparisons of the midline crossing scores among the indicated genotypes. The box plot shows median and 10th, 25th, 75th, and 90th percentiles. Numbers in parentheses indicate the number of scored flies. ${ }^{\star \star \star} p<0.001$ by the Kruskal-Wallis analysis of variance followed by Steel-Dwass nonparametric multiple comparisons. Scale bars: $100 \mu \mathrm{m}$ (a) or $30 \mu \mathrm{m}$ (b). h Male courtship activities. Iola-exon 29 knockdown suppressed male courtship toward a female (3rd bar from the top). Overexpression of wildtype Lola29M (4th bar) or truncation-resistant Lola29M (5th bar) partially rescued the effect of Lola29M knockdown but Lola29F-like (bottom bar) had no rescuing effect. $\mathbf{i}$ Cumulative plot of the number of copulating females as a fraction of time after introducing a wild-type male and a female of the test genotype into an observation chamber. Knockdown by lola-exon 29 RNAi resulted in a reduced copulation success rate ( $30 \%$ ), which was partially rescued by overexpression of Lola29F-like ( $50 \%$ ) but not by overexpression of truncation-resistant Lola29M ( 25\%). j Maximal copulation success in a 1-h observation period. k Courtship indices for partner males used in this experiment. ${ }^{\star \star \star} P<0.001,{ }^{\star} P<0.05$ by the Steel-Dwass post hoc test (h, $\left.\mathbf{k}\right)$ and by the Fisher's exact probability test (j). I Effects of overexpression of 2 different lola transgenes and a fruBM transgene in neurons on the amount of robol mRNA as measured by quantitative RT-PCR. Note that Lola29F-like inhibited the Lola29M action to reduce robo1 mRNA. The numbers of examinations are indicated in parentheses. The box plot shows median and 10th, 25th, 75th, and 90th percentiles. ${ }^{\star \star \star} P<0.001$, ${ }^{\star} P<0.05$ by the one-way ANOVA followed by Bonferroni's multiple comparisons (I)

FruBM-binding region, i.e., the FruBM response obligatory sequence $(\text { FROS })^{13}$, to bind to FruBM (Fig. 5g). We conclude that DR1 is critical for the response of the robol promoter to Lola29M.

To determine whether the observed reduction in robo1 transcript levels by Lola29M overexpression is a result of
Lola29M binding to the robol promoter, we carried out electromobility shift assays (EMSA) for robo1 promoter DNA fragments with V5-tagged Lola29M[K41R] in the absence and presence of unlabeled competitor DNAs (Fig. 5i). We anticipated that Lola29M binds to the DNA fragment carrying FROS (probe DNA B in Fig. 5f). Our EMSA results supported this scenario. A 
a

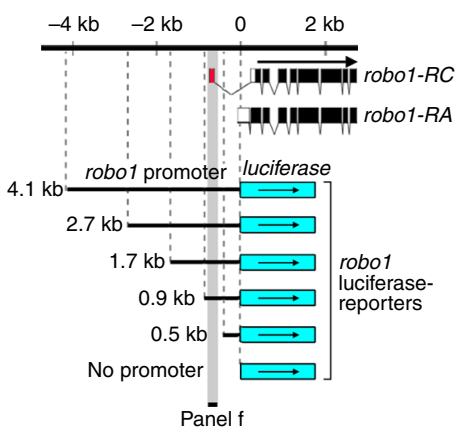

b

$\square$ Mock $(n=5)$
Lola29M $(n=5)$
FruBM $(n=5)$
$\square$ Lola29M and FruBM

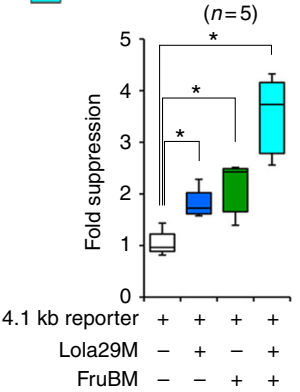

C

$\square \operatorname{Mock}(n=3)$

Lola29M $(n=3)$

Lola29M[K41R] $(n=3)$

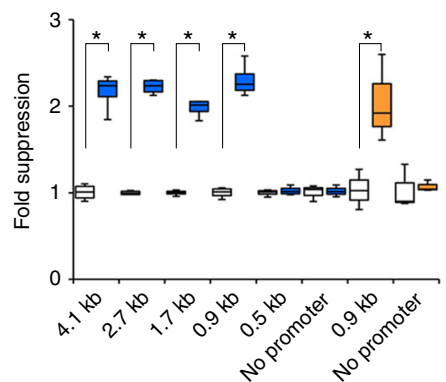

$\mathbf{h} \sqsubset \operatorname{Mock}(n=3)$

Lola29M[K41R]

$(n=3)$

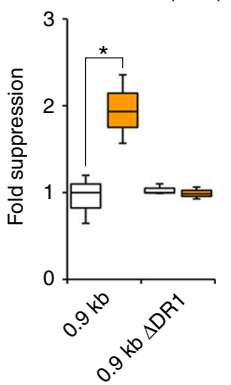

)AT

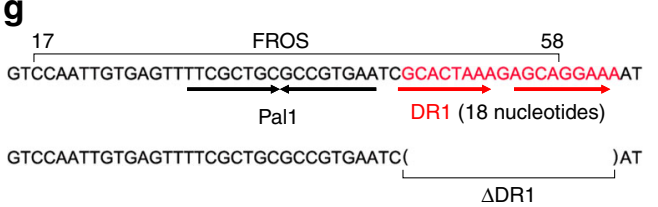

k

j

Probe DNA B +++

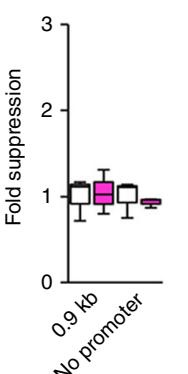
Lola29M[K41R]-V5 +++ Mouse $\lg G-+-$ $\begin{array}{rrrr}\text { Anti-V5 } & \overline{4} & \overline{5} & + \\ & +\end{array}$

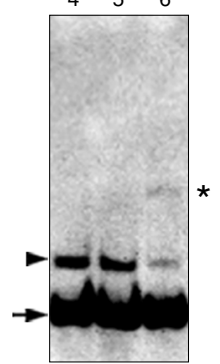

Probe DNA B $\triangle$ DR1 ++ $\begin{array}{lll}\text { Lola29M[K41R]-V5 }- & + & + \\ \text { Unlabeled competitor } & - & \mathrm{B} \Delta \mathrm{DR}\end{array}$ DNA

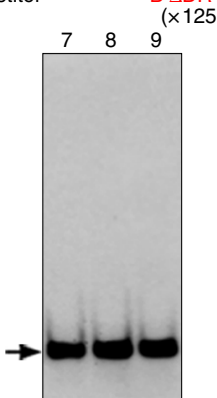

I

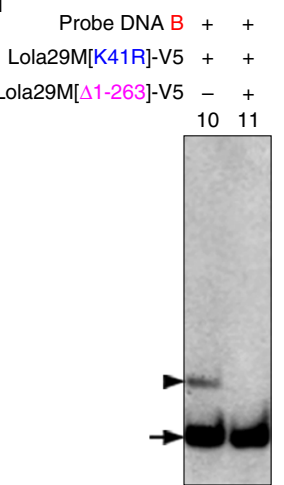

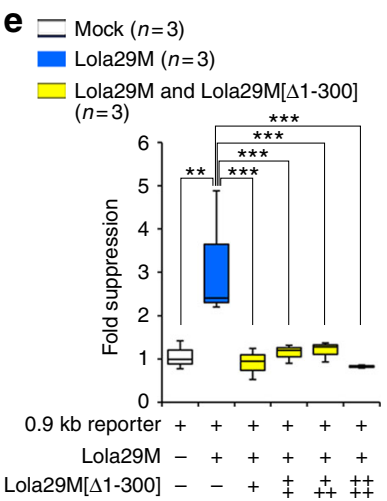

Lola29M[ $[11-300]-\quad-\quad+\quad++++$

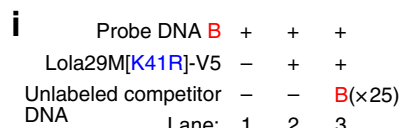

Unlabeled competitor - $-\mathrm{B}(\times 25)$

Lane: $\begin{array}{lll}1 & 2 & 3\end{array}$

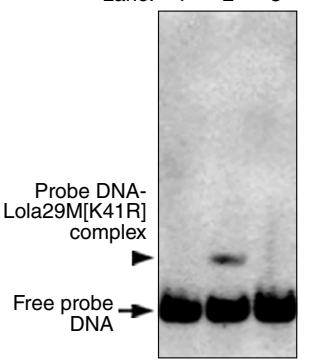

m

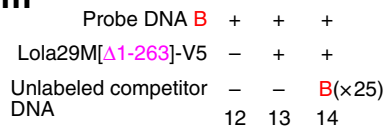

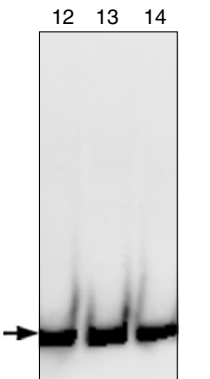

Fig. 5 Lola29M/F molecular actions. a-h robo1 promoter-luciferase reporter assays. a The robo1 genomic map (upper) and reporter schematics (lower). The genomic distance (in $\mathrm{kb}$ ) is indicated on the top with the reference point 0 , at which the robol genomic fragment was fused with the luciferase-coding sequence (blue box with an arrow). Below the thick line, the exon (box)-intron (thin line) organization of robol is shown. Filled and open portions of the box represent the coding and non-coding regions, respectively. $\mathbf{b}$ The relative activities (ordinate) of a $4.1 \mathrm{~kb}$ robol-promoter reporter cotransfected without (-) or with (+) Lola29M, FruBM or both. c The activities of reporters (ordinate) carrying a robo1-promoter fragment of different lengths (the fragment length is indicated on the abscissa) with (filled bars) or without (open bars) cotransfection of Lola29M or truncation-resistant Lola29M[K41R]. d The reporter activities were unaffected by cotransfection with Lola29F-like (Lola29M[ 1 1-300]). e The suppression effect of Lola29M on reporter activities was inhibited by Lola29M[ 1 1-300]. f- $\mathbf{m}$ Lola29M binding to the robo1 promoter. $\mathbf{f}$ Genomic fragments used for EMSA. Probe DNA B and Probe DNA B $\triangle D R 1$ are shown in an expanded scale in the second and third rows. Reporters are schematically illustrated at the bottom. Pal 1: palindrome sequence, FROS: the FruBMbinding region, DR1: direct repeat 1. $\mathbf{g}$ The sequence around DR1 and FROS. The deletion in the $0.9 \mathrm{~kb}$ reporter is also indicated. $\mathbf{h}$ The repressor action of Lola29M[K41R] on the $0.9 \mathrm{~kb}$ reporter with or without DR1. ${ }^{\star} P<0.05$ by the Mann-Whitney $U$ test. $\mathbf{i}-\mathbf{m}$ EMSA with fragment-B detected a retarded band (arrowhead) in the presence of truncation-resistant Lola29M[K41R] (lane 2), which was eliminated by adding the unlabeled fragment-B as competitors (lane 3). Mouse IgG had no effect (lane 5), whereas the Lola29M[K41R]-recognizing anti-V5 induced a super-shift (asterisk; lane 6). The $\Delta D R 1$ probe did not induce retardation (lanes 8). An arrow indicates free DNA. Lola29M failed to produce the retarded band in the presence of Lola29F (lane 11), which on its own, did not produce the retarded band either (lane 13). Source data are provided as a Source Data file

retarded band appeared when Lola29M[K41R] was present (Fig. 5i: lane $2 \mathrm{cf}$. lane 1), which was inhibited by the addition of unlabeled competitive DNA (Fig. 5i: lane 3). The mobility shift was not observed with mouse IgG unrelated to Lola (Fig. 5j: lane 5 cf. lane 4), whereas a super-shift was induced by the anti-V5 antibody that recognizes Lola29M[K41R] (Fig. 5j:* in lane 6). Notably, probe DNA B without DR1 (Probe DNA B $\triangle \mathrm{DR} 1$ ) was unable to produce a retarded band (Fig. 5k). We conclude that 

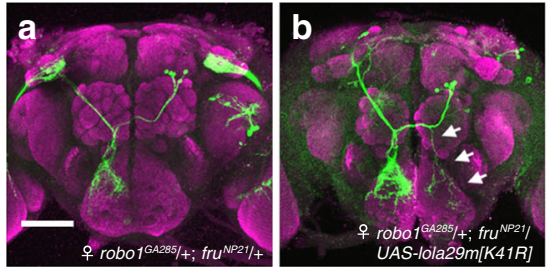

C

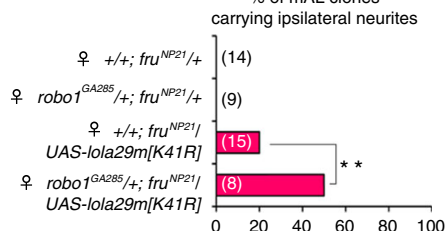

i

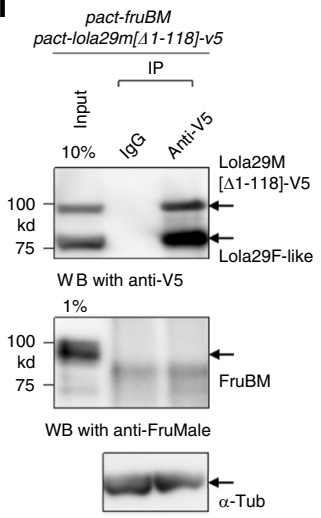

j
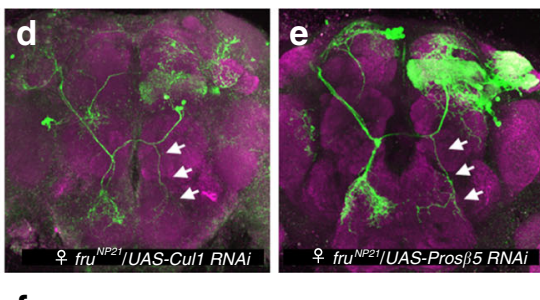

f
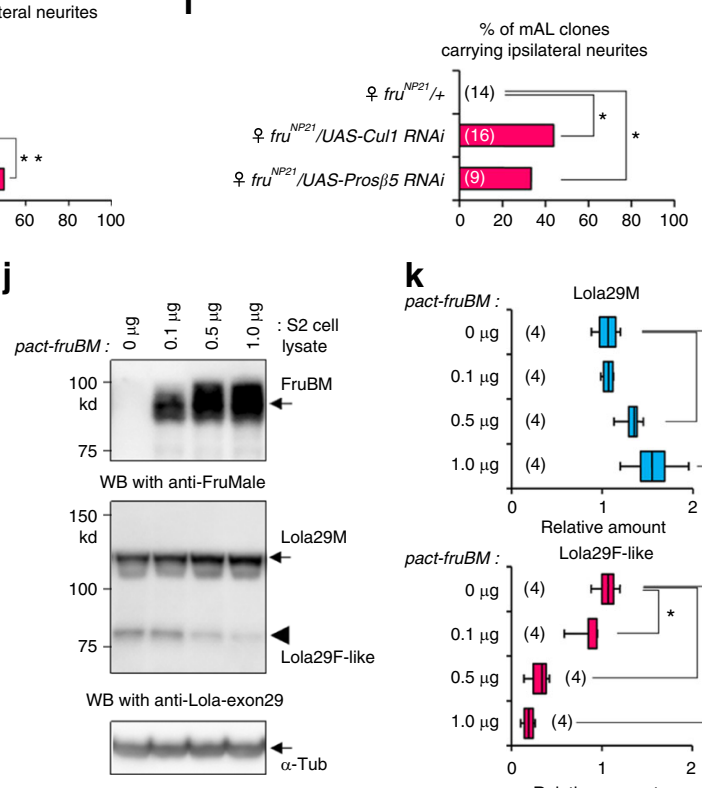

k

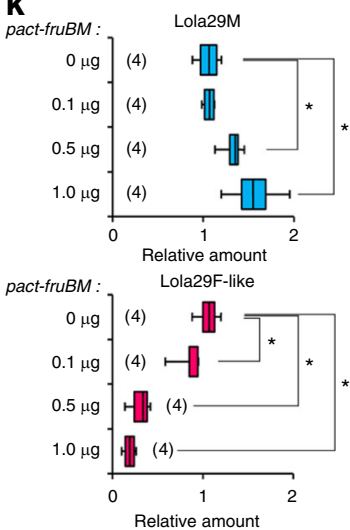

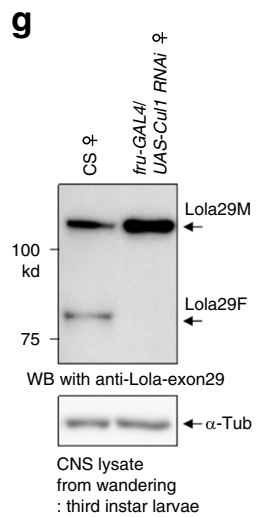
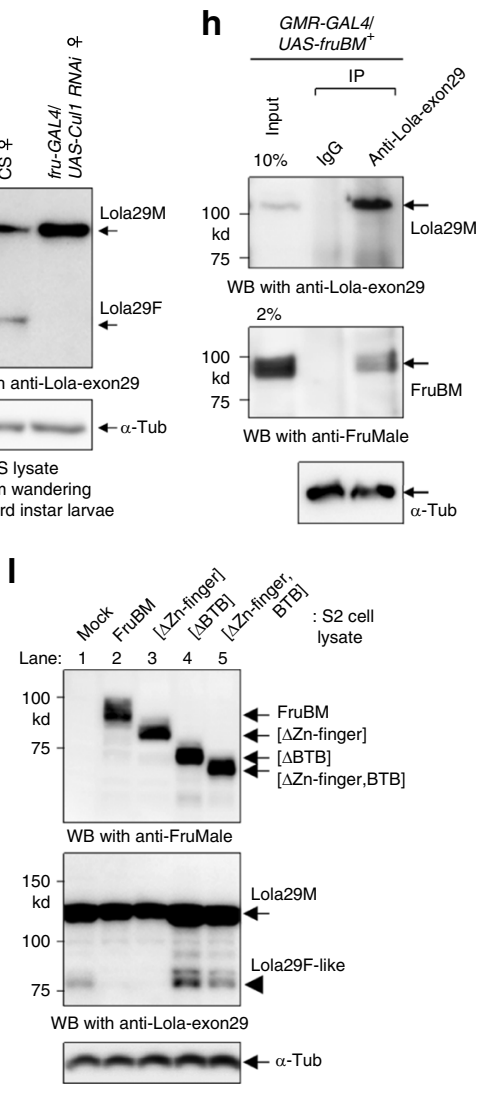

Fig. 6 FruBM protects Lola29M from its ubiquitin-proteasome-mediated truncation. a-c mAL neuroblast clones in robo1GA285/+ females without (a) or with (b) Lola29M[K41R] overexpression and quantitative comparisons of the number of clones carrying the male-specific neurite among the genotypes indicated (c). ${ }^{\star \star} P<0.01$ by the Fisher's exact probability test. d-f Knockdown of Cul1 (d) or Proteasome subunit $\beta 5$ (e) in females results in the formation of the ipsilateral neurite. Representative neuroblast clones with the ipsilateral neurite (d, e) that is otherwise absent from a female neuron. Scale bar: 50 m. f Comparisons of the percentage of $\mathrm{mAL}$ neuroblast clones that carried ipsilateral neurites between females without and with Cul1 or Pros $\beta 5$ knockdown. ${ }^{\star} P<0.05$ by the Fisher's exact probability test. $\mathbf{g}$ Western blotting with anti-Lola-exon 29 of larval CNS extracts with or without Cul1 knockdown. h-I FruBM protects Lola29M from N-terminal truncation. h Overexpressed FruBM is coprecipitated with endogenous Lola29M. Anti-Lola-exon29 was used for

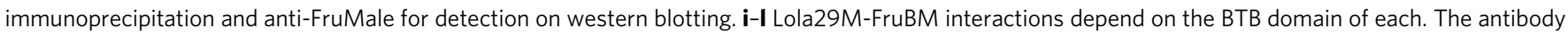
that recognizes the C-terminal V5 tag of Lola29M-V5 (anti-V5) precipitated N-terminally deleted Lola29M-V5 (Lola29M[ 1 1-118]-V5) but not intact

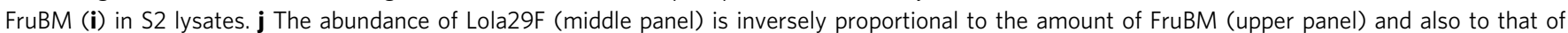
Lola29M (middle panel) in S2 cells. The amount of fruBM-expressing plasmid (pact-fruBM) used for transfection is indicated above each lane. $\alpha$-Tubulin ( $\alpha$ Tub) served as a loading control (lower panel). k Relative abundance of Lola29M (upper panel) and Lola29F-like (lower panel) in the presence of different amounts of FruBM (indicated at the left of the bars). ${ }^{\star} P<0.05$ by the Kruskal-Wallis analysis of variance followed by Steel's nonparametric multiple comparisons. I Deleting the Fru BTB domain resulted in Lola29F production ( $\Delta$ BTB: lane $4 ; \Delta Z n$-finger, BTB: lane 5), whereas deleting the Zn-finger motif ( $\Delta \mathrm{Zn}$-finger: lane 3 ) did not. Western blots of overexpressed proteins probed with anti-FruMale (upper panel), anti-Lola-exon 29 (middle panel) and anti$\alpha$-Tub (lower panel). The box plot shows median and 10th, 25th, 75th, and 90th percentiles

DR1 in the robo1 promoter mediates Lola29M binding. These results collectively suggest that Lola29M directly binds to the robo1 promoter to repress its transcriptional activity. However, Lola29M failed to produce the retarded band in the EMSA with the robo1 promoter fragment in the presence of Lola29F (Lola29M [ $\Delta 1-263]-V 5$; Fig. 5l: lane $11 \mathrm{cf}$. lane 10$)$, which, on its own, did not produce the retarded band either (Fig. $5 \mathrm{~m}$ ). We conclude that Lola29F inhibits Lola29M binding to the robo1 promoter, and consequently derepresses robo1 transcription. DR1 lies just outside FROS and, therefore, is dispensable for FruBM binding to the robo1 promoter ${ }^{13}$. Remarkably, however, male flies homozygous for robo1 ${ }^{\Delta 4}$, a robol mutant carrying a $10 \mathrm{bp}$ deletion that removes DR1, exhibited precocious wing switching during courtship (Supplementary Fig. 11 and Supplementary Movie 1), which represents a behavioral phenotype uniquely observed in flies with defects in the male-specific neurite formation of mAL neurons ${ }^{13}$. For example, robol ${ }^{\Delta 1}$ and robo $1^{\Delta 2}$ mutations that delete a part of the core palindrome Pal1 in FROS dominantly induce the precocious wing switching in male flies ${ }^{13}$. The robo $1^{\Delta 4}$ mutation was recessive in inducing the precocious wing switching and had no dominant effect (Supplementary Fig. 11), suggesting that Lola29M bound to DR1 plays a distinct role that is needed for FruBM to fully repress robo1 transcription.

Next, we examined whether a manipulation of the robol gene dosage could modify the effect of Lola29M in vivo. More specifically, the effect of Lola29M[K41R] overexpression on the male-specific neurite formation was compared between females with the wild-type robol and females heterozygous for a lethal loss-of-function allele, robo1 ${ }^{G A 285}$, which carries a premature termination codon in place of Q411 (http://flybase.org/reports/ FBgn0005631.html). We found that the male-specific neurite was produced by overexpressed lola $29 \mathrm{~m}[\mathrm{~K} 41 \mathrm{R}]$ at a higher rate in the presence of robo1 $1^{G A 285}$ than in its absence (Fig. 6a, b), and the difference was statistically significant (Fig. 6c). Thus, the effect of 
Lola29M[K41R] in promoting the male-specific neurite was enhanced by the robol mutant heterozygosity, which, by itself, has no phenotypic effect. This finding supports the notion that lola and robol interact in vivo.

Lola29M cleavage involves the ubiquitin proteasome pathway. To identify molecules involved in the proteolytic degradation of the Lola29 N-terminus, we analyzed the protein complex that was pulled down with Lola29M in immunoprecipitation assays. We overexpressed Lola29M that lacked a.a. 1-150 (Lola29M[ $\Delta 1$ $150]$ ), as we thought that the N-terminal loss might stimulate further proteolysis of Lola29M. Mass spectrometric analysis identified 121 proteins that were precipitated with Lola29M, including Cul1, an E3 ubiquitin ligase ${ }^{26}$, raising the possibility that this enzyme might be involved in the observed Lola29M truncation (Supplementary Table 1). Expression of Cul1 in mAL neurons was confirmed by the immunostaining with an anti-Cull antibody (Supplementary Fig. 12). We conducted a MARCM analysis in which Cul1 was knocked down in $\mathrm{mAL}$ clones. Remarkably, mAL neurons with Cul1 knockdown led to the malespecific neurite formation in females (Fig. 6d). Moreover, the production of Lola29F was markedly suppressed in females expressing Cul1 RNAi via fru-GAL4 (Fig. 6g). These observations are in keeping with the idea that Cull is involved in the $\mathrm{N}$ terminal truncation of Lola29M.

Ubiquitinated proteins are known to be degraded by the $26 \mathrm{~S}$ proteasome $^{27}$. We thus examined the effect of knocking down Proteasome subunit $\beta 5$ (Pros- $\beta 5$ ), a gene encoding a $26 \mathrm{~S}$ proteasome subunit, on the $\mathrm{mAL}$ structure in the female brain. As shown in Fig. 6e, the expression of Pros- $\beta 5$ RNAi induced formation of the male-specific neurite in female $\mathrm{mAL}$ neurons (Fig. 6f). This observation is compatible with the hypothesis that the ubiquitin-proteasome pathway is involved in the N-terminal truncation and resulting conversion of Lola29M into Lola29F in female $\mathrm{mAL}$ neurons.

FruM inhibits the Lola29M truncation and Lola29F formation. A question arises as to why proteolysis of Lola29M occurs in females but not males. An interesting possibility is that the malespecific protein FruM protects Lola29M against proteolysis. First, we tested the possibility that Lola29M forms a complex with FruM, because both proteins have a BTB domain, through which they may interact directly with each other ${ }^{28}$. As shown in Fig. $6 \mathrm{~h}$, an anti-Lola-exon29 antibody precipitated FruM together with
Lola29M in flies. Moreover, a Lola29M-recognizing antibody failed to precipitate FruM, when the BTB domain of either FruM or Lola29M was deleted in an experiment with cotransfected S2 cells (Fig. 6i and Supplementary Fig. 13). These results support the hypothesis that Lola29M and FruBM form a complex through each other's BTB domains.

Second, we examined the effect of increased FruBM expression on the production of Lola29F in S2 cells transfected with lola29m. FruBM has been shown to be the major FruM isoform that contributes to neural sex differences ${ }^{13,29}$. We found that as the amount of FruBM increased the amount of Lola29F decreased (Fig. 6j, k). Notably, FruBM lost its ability to inhibit the Lola29F production when its BTB domain but not its zinc finger domain was deleted (Fig. 61). These results demonstrate that FruBM binding to Lola29M, presumably as mediated by the BTB domains of two proteins, protects Lola29M against proteolysis, thereby preventing the production of Lola29F in males. Because female neurons lack FruM, Lola29M can be processed into Lola29F.

\section{Discussion}

In this study, we showed that Lola29M, and its truncation product, Lola29F, play important roles in the masculinization and feminization of one of the three types of sexual dimorphism exhibited by fru-expressing mAL neurons: Lola29M promotes the male-specific ipsilateral neurite formation in males, whereas Lola29F counteracts Lola29M and prevents the male-specific neurite from forming in females (Fig. 7). Our results support the hypothesis that males have only the full-length protein Lola29M, because male-specific FruBM binds to Lola29M and protects it from truncation, whereas females have, in addition to full-length Lola29M, N-terminally truncated Lola29F due to the absence of FruBM. As a consequence, the Lola29M action is inhibited by Lola29F in females. This would mean that Lola29M exerts its masculinizing action only when FruBM is present, representing an efficient and secure means to induce masculine characteristics in fru-expressing neurons.

Our EMSA results demonstrated that Lola29M and FruBM share the same transcriptional target gene robo1, the transcription of which is repressed by each protein or by both proteins in synergy, according to our reporter assay data. The robo1 gene encodes a transmembrane receptor of the immunoglobulin superfamily, Robo1 ${ }^{30}$, which is a key effector to prevent the male-specific ipsilateral neurite from forming in
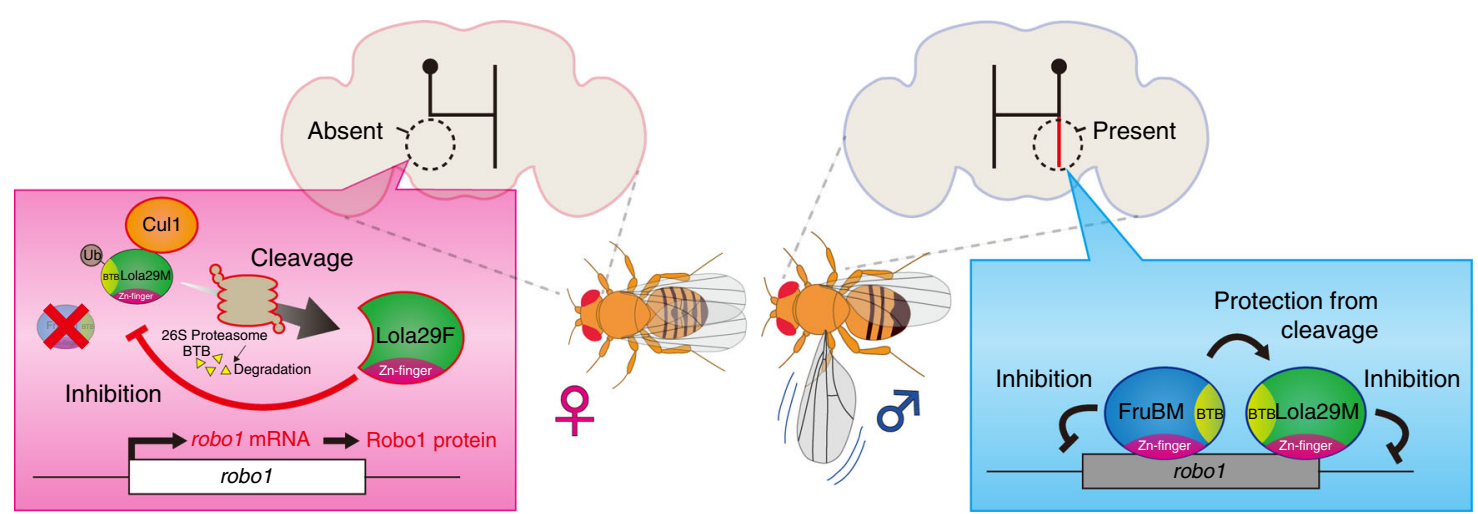

Fig. $7 \mathrm{~A}$ model of the interaction between Lola29M and Fru in shaping the sexual dimorphism of mAL neurons and fly sexual behavior. Female neurons (left-hand side) lack FruBM, and consequently Lola29F is produced in sufficient abundance to impede the masculinizing action of Lola29M, leading to the production of mAL neurons without the ipsilateral neurite. Male neurons (right-hand side) express FruBM, which forms a complex with Lola29M and represses proteolysis of Lola29M. This results in the accumulation of Lola29M, which induces the ipsilateral neurite in some mAL neurons via robo1 repression 
females ${ }^{13}$. Lola29M induces the male-specific ipsilateral neurite by transcriptional repression of robol (acting as a negative regulator of neuritogenesis) in males, whereas Lola29F appears to compete with Lola29M, allowing robo1 to be transcribed, and as a result, Lola29F blocks the male-specific neurite formation in females. Our EMSA data identified two distinct DNA stretches, one harboring a Lola29M-binding site and the other a FruBM-binding site ${ }^{13}$ in the robo1 promoter region, which were located side-by-side. On the other hand, our immunoprecipitation assays indicated that Lola29M and FruBM form a complex in vivo. It might be that Lola29M and FruBM each bound to their own binding sites interact in trans, leading the robol promoter to a conformation unfavorable for transcriptional activation.

We showed that the replacement of four lysine residues, K41, K44, K47 and K67, which are potential ubiquitination-targets, with arginine residues conferred resistance to N-terminal truncation on Lola29M, suggesting that the ubiquitin proteasome pathway is involved in the Lola29M processing. We also showed that the E3 ubiquitin ligase Cull is required for the Nterminal truncation of Lola29M, and that the male-specific neurite formation is induced by Cull knockdown in female mAL neurons. In natural killer T-cell thymocytes, the E3 ubiquitination enzyme Cul3 is recruited to a chromatin-modifying complex, where it then induces changes in the ubiquitination patterns of other components of the same complex ${ }^{26}$. This recruitment of $\mathrm{Cul} 3$ to the chromatin-modifying complex is mediated by BTB zinc finger proteins such as PLZF and $\mathrm{BCL}^{26}$. Therefore, it may be possible that Lola29M truncation is similarly mediated by a component in the protein complex to which Lola29M contributes. In this context, it would be worth mentioning that Cul1 and Cul3 play contrasting roles in the regulation of hedgehog ( $h h$ ) signaling; in the absence of $\mathrm{Hh}$, Cull is recruited to the Hh downstream zinc finger protein Cubitus interruptus (Ci155), which undergoes, as a consequence, partial degradation to yield the transcriptional repressor Ci75, whereas upon the $\mathrm{Cul} 3$ recruitment in the presence of $\mathrm{Hh}, \mathrm{Ci} 155$ is completely degraded ${ }^{31}$. It remains to be determined how Cul1 is recruited to Lola29M for its partial degradation in fru-positive neurons.

A recent study documented neural activity-dependent activation of lola transcription, leading to the proposition that lola is an immediate early gene in insects ${ }^{32}$. The transient expression common to immediate early gene products is caused by the rapid degradation that follows their rapid induction. An intriguing possibility is that Lola proteins are vulnerable to rapid degradation through ubiquitination, unless their ubiquitination target sites are masked by a binding partner, such as FruBM, that interacts through the BTB domain. The known pleiotropic functions of Lola invite the supposition that other Lola isoforms expressed in a variety of cell types might form a complex with the different BTB proteins expressed there, accomplishing diversified roles unique to the individual cell type.

The present work thus provides new insights into the molecular mechanism whereby a common transcription factor such as Lola can play distinct roles in different cells in a contextdependent manner. A growing body of evidence indicates that fate changes in a variety of cell lineages in both vertebrates and invertebrates are induced by rapid epigenetic remodeling by chromatin regulators, such as BTB-zinc finger transcription factors, some of which are known to recruit E3 ubiquitin ligases to the chromatin-modifying complex and thus alter its ubiquitination pattern. It is therefore plausible that the proteolytic removal of the N-terminal BTB domain from these transcription factors within the chromatin-modifying complex is a prevalent means to convert the epigenetic marks.
Moreover, there is accumulating evidence that the association of BTB proteins with ubiquitin ligases plays multilayered regulatory roles in developmental decisions. For example, Germ cellless $(\mathrm{Gcl})$, a BTB protein conserved from C. elegans to humans with a transcriptional repressor activity ${ }^{33,34}$, plays a key role in the soma-germ fate switch via a non-transcriptional mechanism. When complexed with Cul3, Gcl exits the nucleus to degrade the somatic fate determinant Torso and promotes the primordial germ cell fate in Drosophila ${ }^{35}$. In vertebrates, the Cul3-KBTB18 complex switches the translational program so as to specify the neural crest fate ${ }^{36}$. The present study further expanded the roles of the ubiquitin proteasome system-namely, the system was shown to function in the specification of sex-types of neurons via regulated proteolysis of a transcription factor that functions in the sex-determination molecular machinery.

\section{Methods}

Fly strains. Flies were reared on cornmeal-yeast medium at $25^{\circ} \mathrm{C}$. Canton-S served as a wild-type control. The following $f r u$ alleles were used: $f r u^{s a t}, f r u^{2}$, and $f r u^{N P 21}$ The GAL4 driver $f r u^{N P 21}$ used in this study is a recessive allele of fru induced by a P-element insertion into the fru second intron (Supplementary Fig. 1a), which exhibited no discernible defect in neurite structures. A subset of mAL neurons lacked the ipsilateral neurite in $f \mathrm{ru}^{N P 21} /+$ heterozygous males, as were the case in males of some flylight lines 37 , which carried driver GAL4 insertions at genomic sites unrelated to the fru locus ${ }^{3}$. The GS2169 strain (\#200307) carrying a GS vector inserted into the 5' region of the lola gene was obtained from the Drosophila Genetic Resource Center (Kyoto, Japan). elav-Gal4C155 (elav-Gal4, \#458), robo1 ${ }^{\text {GA285 }}$ (\#8755), tra ${ }^{1}$ (\#675), UAS-Cul1-RNAi (\#29520), UAS-Prosß5 RNAi $(\# 34810)$ and other fly resources used in MARCM were obtained from the Bloomington Drosophila Stock Center. The UAS-lola-COM RNAi (\#12573 and \#12574) and UAS-lola-exon29 RNAi (\#25335) lines were obtained from the Vienna Drosophila RNAi Center. The following fly lines were generous gifts from the indicated researchers: lolaORE76 (an EMS-induced strong allele ${ }^{18}$, Drs. M. L. Spletter and L. Luo); lola ${ }^{03089}$ (a transposon-inserted hypomorphic allele ${ }^{18}$, Dr. T. Aigaki); inscGAL4 (Dr. A. Knoblich); fru-GAL4, 9-189-GAL4, UAS >stop >mCD8::GFP and $f r u^{F L P}$ (Dr. B. Dickson); poxn-GAL4 (Dr. K.-i. Kimura).

Modifier screens. The female flies with both GMR-GAL4 and UAS-fru-typeB ${ }^{+}$ (denoted as $U A S$-fruB ${ }^{+}$in Supplementary Fig. 2) transgenes were crossed with male flies from GS P-element insertion stocks ${ }^{15}$ or those from mutant stocks reported to have developmental defects in the nervous system (FlyBase: https:/ flybase.org/). In this screen, we overexpressed a FruCOM protein rather than a FruM protein when inducing the rough eye phenotype, as the former yielded more viable offspring. The nomenclature for Fru isoforms is adapted from our previous study $^{38}$ and different from that of other groups ${ }^{39} .1364$ stocks were screened, resulting in 40 dominant suppressors of the rough-eye phenotype induced by frutype $B^{+}$overexpression. Among the 5 Fru C-terminal variants, TypeB was most effective in rescuing the $\mathrm{fru}^{\text {sat }}$ mutant phenotype $\mathrm{e}^{38}$ and thus was most likely to yield modifiers that were relevant to the in vivo functions of fru. Images of the compound eye surface were obtained with a scanning electron microscope (SU8000; Hitachi High-Technologies, Tokyo, Japan).

Plasmids and transformants. lola $29 \mathrm{~m}$ cDNAs with or without an amino acid replacement (point mutants include: $K 41 R, K 44 R, K 47 R, K 67 R, K 74 R, K 77 R$, K105R, K113R, K127R, K138R, K149R, K181R, K255R, K256R, and K261R; Fig. 2f) or with N-terminal deletions (deletants include: $\Delta 1-118, \Delta 1-150$ and $\Delta 1-300$ ) were attached by the sequence for the N-terminal HA tag, and cloned into the $p M T / V 5$ $H i s \mathrm{C}$ vector (Invitrogen). Subsequently, transgenic fly lines were generated with some of these constructs, i.e., HA-lola29m[K41R]-V5 (Fig. 2g), HA-lola29m[D1300]-V5 (Fig. 1f) and HA-lola29m-6xV5 (Fig. 2a), which were cloned into a pJFRC81 vector ${ }^{40}$ having a GFP coding sequence replaced by the respective lola $29 \mathrm{~m}$ CDNA sequence. The constructs thus generated were integrated into the attP2 site of the D. melanogaster genome using commercial microinjection services (BestGene and Genetic Services). To construct pact-FLAG-fruBM[ $\triangle B T B]$ and pactFLAG-fruBM $[\triangle Z$ inc, $B T B]$ vectors (denoted as $\triangle B T B$ and $\triangle Z n$-finger, $B T B$ in Fig. 6l), 111 amino acids (QQFCLRWNNHPTNLTGVLTSLLQREALCDVTLACEGETVKAHQTILSACSPYFETIFLQNQHPHPIIYLKDVRYSEMRSLLDFMYKGEVNVGQSSLPMFLKTAESLQVRGL) composing the BTB domain were deleted from pact-FLAG-fruBM ${ }^{9}$ or pact-FLAG-fruBM[DZinc $]^{13}$ respectively. To obtain pact-lola29m-v5 and pact-lola29m[ $\Delta 1-118]-v 5$ vectors (Figs. 5b and 6i), cDNA encoding lola29m- $v 5$ or lola29m[ $\Delta 1-118]$-v5 was amplified from aforementioned $p M T$ vectors and cloned into the pact-MCS vector ${ }^{9}$.

Antibody production. A rabbit polyclonal anti-Lola-exon 29 antibody (Fig. 1c) was raised against a mixture of two 19-mer peptides, HARQEYIKIDTSRLEDKML and YRSDLRKHMNQKHADSGEA, which were both encoded by exon 29 of the 
lola gene (residues 582-600 and 749-767 of Lola isoform Q (GenBank accession number AB107288)), and was affinity purified. A rabbit polyclonal anti-LolaCOM antibody (Supplementary Fig. 6) was raised against an 18-mer peptide, GGVAPKPESSGHHRGGKC, which was situated 4 amino acids distal to the BTB domain, within a region conserved in all isoforms of the Lola protein (residues 122138 of the Lola proteins), and was affinity purified. The guinea pig polyclonal antiFruMale antibody (Fig. 1i) was generated against a 19-mer peptide,

HYAALDLQTPHKRNIETDV, in the male-specific N-terminus of Fru (residues 56-74 of FruM), and was affinity purified.

Western blot assays. For evaluating the specificity of the anti-Lola-exon 29 antibody (Supplementary Fig. 3), whole-cell extracts were prepared from lola heterozygous (lola ORE76/CyO-GFP) or homozygous (lolaORE76/lola ORE76) embryos at developmental stages 11-14 by homogenizing them in a $4 \mathrm{x}$ sample buffer solution (Wako, 198-13282) containing $200 \mathrm{mM}$ DTT. To determine the lolaknockdown effect by UAS-lola-COM RNAi or UAS-lola-exon29 RNAi (Supplementary Fig. 6), eye-antennal discs isolated from ten 3rd instar larvae were homogenized in the sample buffer. For the analysis of expression patterns of Lola proteins at the wandering 3rd instar larval stage (Fig. 1c), extracts were prepared by homogenizing the central nervous system from sexed individuals: males and females of Canton-S wild-type, males of $f_{r u} u^{\text {sat }} f r u^{N P 21}$ (selected as those not carrying a fluorescent balancer, TM3 $\mathrm{Sb} \mathrm{Kr}$-Gal4 UAS-GFP), females of $\mathrm{tra}^{1}$ homozygotes (selected as those not carrying a fluorescent $\mathrm{Y}$-chromosome balancer, $Y$ $G F P+{ }^{41}$, or males and females of the indicated genotypes (Fig. 1c) in the sample buffer. For Western blot analysis of S2 cell lysates (Fig. 2c, d, f), cells were disrupted by incubation with a lysis buffer (50 mM HEPES, pH 7.5, $300 \mathrm{mM} \mathrm{NaCl}, 50 \mu \mathrm{M}$ $\mathrm{ZnSO}_{4}, 10 \mathrm{mM} \mathrm{NaF}, 0.4 \%$ Nonidet P-40 (NP40) and cOmplete Protease Inhibitor (Roche)) for $1 \mathrm{~h}$ at $4{ }^{\circ} \mathrm{C}$. Lysates were prepared by centrifugation and denatured in the sample buffer. After fractionation by SDS-PAGE and transfer to PVDF membranes (Life Technologies, IB401001), the blots were reacted with an antibody, rabbit anti-Lola-exon 29 (1:100; present study), mouse anti-V5 (1:500; Invitrogen, 46-0705), rat anti-HA (1:500; Roche, 11867423001), rabbit anti-FruMale $(1: 500)^{38}$, rabbit anti-LolaCOM (1:200; present study), or mouse anti- $\alpha$-Tubulin (1:500; Sigma, T6199), and subsequently by a horseradish peroxidase (HRP)-conjugated anti-rabbit, rat, or mouse IgG antibody (1:3,000; Sigma). Chemiluminescence was detected with Pierce Western Blotting Substrate Plus (Thermo Scientific, NCI32132) according to the manufacturer's instructions. Fluorescent images were obtained using ImageQuant LAS 4000 (Fujifilm). The signal intensity was quantified by using the software package ImageQuant TL (GE Healthcare). The endogenously expressed Fru proteins may affect transfection assays in S2 cells. We performed western blot analysis with S2 lysates for FruM expression several times, detecting no endogenous FruM expression (Supplementary Fig. 14). Even if Fru is expressed in S2 cells, the S2-derived Fru is likely non-sex-specific FruCOM rather than FruM, because the S2 cells originated from non-neural cells (probably lymphocytes), while FruM is specifically expressed in neural cells of males. We were unable to determine whether FruCOM is expressed in S2 cells due to the lack of an anti-FruCOM antibody that works in western blotting.

Coimmunoprecipitation assays. In the co-immunoprecipitation assays for Lola and Fru (Fig. 6h), we overexpressed frut-type BM in developing eye discs with the GMR-Gal4 driver, as endogenous Fru proteins are expressed only in $\sim 2 \%$ of neurons in the male nervous system and thus it is difficult to recover a sufficient quantity for analysis. CNS/eye-antennal disc complexes of wandering $3 \mathrm{rd}$ instar larvae were collected and homogenized in a cold lysis buffer (50 mM HEPES, $\mathrm{pH}$ 7.5, $300 \mathrm{mM} \mathrm{NaCl}, 50 \mu \mathrm{M} \mathrm{ZnSO}_{4}, 10 \mathrm{mM} \mathrm{NaF}, 0.4 \% \mathrm{NP} 40$ and cOmplete Protease Inhibitor (Roche)) for $1 \mathrm{~h}$ at $4{ }^{\circ} \mathrm{C}$. Lysates were prepared by centrifugation and precleared with protein G beads (GE Healthcare, 17061801). After removal of the beads, the lysate was incubated with rabbit IgG (Sigma, I5006) or the anti-Lola-exon29 antibody in the aforementioned lysis buffer, which was modified to contain $150 \mathrm{mM} \mathrm{NaCl} / 0.2 \% \mathrm{NP} 40$, for $3 \mathrm{~h}$ at $4^{\circ} \mathrm{C}$. The immuno-complexes were precipitated using protein G beads (GE Healthcare, 17061801) and analyzed by Western blotting with a primary antibody, i.e., rabbit anti-FruMale $(1: 500)^{38}$, rabbit anti-Lola-exon29 (1:100; present study), or mouse anti- $\alpha$-Tubulin (1:500; Sigma T6199), and, as a secondary antibody, horseradish peroxidase (HRP)-conjugated anti-rabbit or mouse IgG (Sigma). In the assays shown in Fig. 6i and Supplementary Fig. 13, FruBM and Lola29M proteins with or without the indicated deletion ( $\triangle \mathrm{BTB}$ in FruBM, $\Delta 1-118$ in Lola29M) were co-overexpressed in S2 cells. $1 \mu \mathrm{g}$ of each of the expression vectors was transfected into S2 cells ( $1 \times 10^{7}$ cells) using FuGene HD Transfection Reagent (Promega, E2311). Forty-eight hours after transfection, lysates were prepared by homogenizing in the aforementioned cold lysis buffer, then incubated with mouse IgG (Invitrogen, 10400C) or mouse anti-V5 antibody (Invitrogen, R96025 ) in the lysis buffer with $150 \mathrm{mM} \mathrm{NaCl}$ and $0.2 \% \mathrm{NP} 40$ for $3 \mathrm{~h}$ at $4{ }^{\circ} \mathrm{C}$. The immuno-complexes were precipitated using protein G beads (GE Healthcare, 17061801 ) and analyzed by Western blotting with a primary antibody, i.e., mouse anti-V5 (1:500; Invitrogen, R960-25) or rabbit anti-FruMale (1:500) ${ }^{38}$ and, as a secondary antibody, horseradish peroxidase (HRP)-conjugated antimouse or rabbit IgG (Sigma).
Immunohistochemistry. Immunostaining was carried out as described previously ${ }^{9}$. For immunostaining of the adult brain (Fig. 3d-g), the brains of 2- to 7 day-old flies of the relevant genotypes were dissected in phosphate-buffered saline (PBS), and fixed in 4\% paraformaldehyde in PBS for $60 \mathrm{~min}$ on ice. After washing in PBT (PBS with $0.3 \%$ Triton X-100), the tissues were kept in PBTN (PBT with $10 \%$ normal goat serum) for $60 \mathrm{~min}$ on ice for blocking, and reacted with the primary antibody in PBTN for $24 \mathrm{~h}$ at $4^{\circ} \mathrm{C}$. After $2 \mathrm{~h}$ of washing in PBT, the tissues were incubated in the secondary antibody for $24 \mathrm{~h}$ at $4{ }^{\circ} \mathrm{C}$. Samples were washed for $2 \mathrm{~h}$ in PBT before mounting with Vectashield (Vector Laboratories Inc.). To immunostain eye-antennal imaginal discs (Supplementary Fig. 2b) and the CNS (Supplementary Fig. 4 and Supplementary Fig. 10) of third instar larvae, the discs and CNS of relevant genotypes were dissected in PBS and fixed in $4 \%$ paraformaldehyde for $60 \mathrm{~min}$ on ice, and subsequent immunostaining was performed following the method described above. The antibodies used were as follows: rabbit polyclonal anti-GFP (1:500; Invitrogen A6455), mouse nc82 (1:10; Hybridoma Bank), guinea pig anti-FruMale (1:500; present study), rabbit anti-FruCOM $(1: 500)^{38}$, rat anti-Deadpan (1:100; Abcam, ab195173), mouse anti-Prospero (1:20, Hybridoma Bank), rabbit anti-Lola-exon29 (1:100; present study), mouse antiRobol 13C9 (1:50, Hybridoma Bank), chicken anti-GFP (1:500, abcam ab13970), rabbit anti-Cul1 (1:100, Invitrogen 71-8700) and secondary Alexa-488, 546, and 647 antibodies (1:500; Invitrogen). Stacks of optical sections at $1 \mu \mathrm{m}$ were obtained with a Zeiss LSM 510 META confocal microscope and processed with NIH Image J (http://rsb.info.nih.gov/ij/) and Adobe Photoshop software.

Clonal analysis of mAL neurons. We used $\mathrm{fru}^{\mathrm{NP21}}$-GAL4 to label mAL neurons (Fig. $1 \mathrm{~m}-\mathrm{p}$ ). The somatic clones were produced using the MARCM method ${ }^{42} . y \mathrm{hs}-$ flp / Y; FRT G13 UAS-mCD8-GFP / FRT G13 tub-Gal80; fru ${ }^{\text {sat } / ~ f r u}{ }^{N P 21}$ was used for the fru mutant males (referred to as $f r u^{\text {sat }} / f_{r u} u^{N P 21}$ in Fig. 3d). The genotypes of flies used for the clonal expression of Lola29M[K41R] or Lola29M[ $[\Delta 1-300]$ in fru mutant males (Fig. 3e-g) were as follows: $y$ hs-flp / Y; FRT G13 UAS-mCD8-GFP / FRT G13 tub-Gal80; frusat / fru NP21 UAS-HA-lola29m[K41R]-V5-6xHIS or fru ${ }^{N P 21}$ UAS-HA-lola29m[S1-300]-V5-6xHIS. For clonal expression of Lola29M[K41R] or Lola29M $[\Delta 1-300]$ in fru heterozygous flies (Fig. 3a, b and Supplementary Fig. 9), flies of the following genotypes were used: $y$ hs-flp / $w$ (female) or $Y$ (male); FRT G13 UAS-mCD8-GFP / FRT G13 tub-Gal80; fru ${ }^{\mathrm{NP} 21} /$ + (control referred to as $f_{r u}{ }^{N P 21} /+$ in Supplementary Fig. 9a) or UAS-HA-lola29m[K41R]-V5-6xHIS (Lola29M[K41R] overexpression, Supplementary Fig. 9b) or UAS-HA-lola29m[ $\Delta 1$ 300]-V5-6xHIS (Lola29M[ $\Delta 1-300]$ overexpression, Supplementary Fig. 9c). The genotype of flies used in clonal Lola29 knockdown experiments (Fig. 1o, p) was: $y$ hs-flp / Y; FRT G13 UAS-mCD8-GFP / FRT G13 tub-Gal80; fru NP21 / UAS-lolaexon29 RNAi (VDRC\#25335). The genotype of flies used in clonal co-expression of Lola29M and Lola29F-like (Fig. 3g) was: $y$ hs-flp / Y; FRT G13 UAS-mCD8-GFP / FRT G13 tub-Gal80; fru NP21 UAS-ha-lola29m[41-300]-v5-his / fru sat UAS-halola29m[K41R]-v5-his. The genotype of flies used in clonal Cul1 and Pros $\beta 5$ knockdown experiments (Fig. 6d, e) was: $y$ hs-flp / w; FRTG13 tub-Gal80 / FRTG13 UAS-mCD8-GFP; fru ${ }^{N P 21} /$ UAS-Cul1 RNAi (BL\#29520) or UAS-Pros $\beta 5 R N A i$ (BL\#34810). The genotype of flies used in clonal expression of Lola29M[K41R] in robo1 heterozygous females (Fig. 6a, b) was: $y$ hs-flp UAS-mCD8-GFP / w; FRT40A tub-Gal80 / robo1 ${ }^{\text {GA285 FRT40A; fru }}$ NP21 / + (control, panel a) or UAS-ha-lola29m [K41R]-v5-his (Lola29M overexpression, panel b). For the production of neuroblast clones in mAL neurons, embryos at 0 to $24 \mathrm{~h}$ after egg laying (AEL) were heat shocked at $37^{\circ} \mathrm{C}$ in a water bath for $1 \mathrm{~h}$. For the production of single-cell clones of $\mathrm{mAL}$ neurons, larvae at $5-6$ days AEL were heat shocked at $37^{\circ} \mathrm{C}$ for $1 \mathrm{~h}$. Flies to be tested were reared at $29^{\circ} \mathrm{C}$ after the heat shock in order to enhance the expression of transgenes. The number of clones examined was different from experiment to experiment as a consequence of the stochastic nature of mosaic generation. This might complicate interpretations of data, particularly in the experiment shown in Figs. $3 c, 6 c$ and $6 f$, where the proportion of neurons with the ipsilateral neurite was quantitatively compared. We therefore performed a post hoc power analysis of these data with the aid of Webpower ${ }^{43}$, which yielded the following results: Power $=0.7194, \alpha=0.05$ for Fig. 3c; power $=0.3095, \alpha=0.05$ for Fig. $6 c$; power $=0.9774, \alpha=0.05\left(f^{2} u^{N P 21} /+v\right.$ s. $\left.f u^{N P 21} / U A S-C u l 1 R N A i\right)$ and power $=$ $0.8209, \alpha=0.05$ (fru NP21 $^{\text {N }}+$ vs. $\left.f r u^{N P 21} / U A S-P r o s-\beta 5 R N A i\right)$ for Fig. 6 . Here, the sample number is considered to be large enough when it gives a power value larger than 0.7. The data shown in Figs. $3 \mathrm{c}$ and $6 \mathrm{f}$ fulfilled this criterion but those in Fig. $6 c$ did not. Our calculations indicate that power values over 0.7 would be obtained if 23 or more samples were subjected to analysis in the experiment shown in Fig. $6 c$ ( $c f .8-15$, the current sample numbers). However, the flies with the genotype robo1 $1^{G A 285} /+$; fru NP21/UAS-lola29M ${ }^{K 41 R}$ were extremely difficult to obtain as most of the individuals died before eclosion. Because the statistical difference at $P<0.01$ found in this set of data stands regardless of whether the power exceeds 0.7 or not, we consider that the result in Fig. 6c supports our conclusion that robo1 and lola (Lola29M) interact in vivo.

Midline crossing score analysis. The MCS (Fig. 4g) was calculated as described previously ${ }^{9}$. Briefly, stacked images of each sample were summed up using ImageJ, and a circle of $8 \mu \mathrm{m}$ (marked as "a" in Fig. 4f) was drawn on the resultant image so that the circle was centered at the prothoracic midline, where trans-midline axons are expected to run in males. The fluorescent intensity within the circle marked "a" was measured to quantify the level of midline crossing by fibers. Similarly, the fiber 
tracts locating lateral to the midline were quantified within circles "b1" and "b2" for the fluorescent intensity. To normalize for the background fluorescent level, the areas with no fibers delineated by circles "c1" and "c2" were also measured. The MCS was calculated as: MCS $=[a-(c 1+c 2) / 2] /[(b 1+b 2) / 2]$.

Behavioral assays. The flies to be tested were reared individually in vials under a $12: 12 \mathrm{~h}$ light:dark cycle. For analysis of male courtship behavior (Figs. 1a, b, $4 \mathrm{~h}$ and Supplementary Fig. 11), virgin males of the indicated genotypes were collected at eclosion and aged for 5-7 days. Canton-S virgin females were similarly prepared as mating partners of test males. In the behavioral assays, a male of each genotype and a Canton-S virgin female were paired in a small chamber of $8 \mathrm{~mm}$ in diameter and $3 \mathrm{~mm}$ in height. The flies were video recorded for $5 \mathrm{~min}$. The courtship index (CI) was determined as the percentage of time that the male courted the females during a 5 min observation period. In calculating the CI, the time spent for all courtship elements, i.e., orientation, tapping, following, wing extension/vibration and attempted copulation, was included. The wing switching index was estimated by the method as described in ref. ${ }^{13}$. For the analysis of female mating behavior (Fig. 4i-k), virgin females of each genotype were collected at eclosion and aged for 5-7 days. Each female fly was transferred to a small chamber $(8 \mathrm{~mm}$ in diameter and $3 \mathrm{~mm}$ in height) with a Canton-S virgin male. The behavior of the fly pair was recorded using a video recorder. To estimate the level of female sexual receptivity, the cumulative number of copulating pairs in a $1 \mathrm{~h}$ observation period was counted and compared among the fly groups of different genotypes.

S2 cell culture. Drosophila S2 cells (Invitrogen, R690-07) were grown in Schneider's Drosophila medium (Gibco, 21720-024) supplemented with 10\% fetal bovine serum (HyClone, SH30071.03, heat-inactivated) and 1\% antibiotic-antimycotic solution (Gibco, 15240062). To determine the effect of proteasome inhibitors (Fig. $2 \mathrm{c}, \mathrm{d}$ ), approximately $2 \times 10^{6}$ cells were prepared in triplicate for transfection with a lola29m expression vector: the first set was treated with MG-132 (InvivoGen, tlrl-mg132, affinity purified and dissolved in DMSO) at concentrations of 0-50 $\mu \mathrm{M}$ for $5 \mathrm{hr}$, the second set was treated with Lactacystin (Kyowa Medex, OP18, dissolved in ethanol) at concentrations of $0-20 \mu \mathrm{M}$ for $6 \mathrm{~h}$ and the third set was left without drug administration. $0-1 \mu \mathrm{g}$ of pact-fruBM (Fig. 6j) ${ }^{9}, 1 \mu \mathrm{g}$ of pact-fruBM with or without the indicated modifications (Fig. 6l), or $1 \mu \mathrm{g}$ of modified $P M T-H A-$ lola29m-V5 (Fig. 2f) was transfected into approximately $1 \times 10^{7}$ cells per plate by using FuGENE HD Transfection Reagent (Promega, E2311). Forty-eight hours after transfection, the cells were disrupted by incubation with the aforementioned lysis buffer for $1 \mathrm{~h}$ at $4{ }^{\circ} \mathrm{C}$ and subjected to the analysis of expression profiles of Lola protein isoforms.

RACE PCRs. 5' and 3' RACE PCRs (Supplementary Fig. 5) were performed using the SMARTer RACE $5^{\prime} / 3^{\prime}$ kit (Clontech, Z4858N). Poly A(+) RNA was extracted from the CNS of a wandering third instar larva of a Canton-S female using the Micro-FastTrack 2.0 kit (Life Technologies, K1520-02). RACE-ready cDNA was synthesized from the extracted RNA. For 5' RACE, the forward primer was a mixture of oligo named " 5 ' RACE primer (F)", which was used in conjunction with the reverse primer named "Primer 1 (R)", a gene-specific primer. For 3' RACE, the forward primer was the gene-specific "Primer $2(\mathrm{~F})$, which was used in conjunction with the " 3 RACE primer (R)", a mixture of oligo. For 3 " and 5' RACE PCR, the following gene-specific primers were used: Primer 1 (accatccagcaatcgcagacgcatgc) and Primer 2 (cgcaggagcatcttgtcctccagcct), respectively. The cycling parameters for 3 ' and 5' RACE-PCR were as follows: 1 cycle of $94^{\circ} \mathrm{C}$ for $2 \mathrm{~min}, 30$ cycles of $94^{\circ} \mathrm{C}$ for 30 seconds and $63^{\circ} \mathrm{C}$ for 30 seconds and $68^{\circ} \mathrm{C}$ for $2 \mathrm{~min}$, followed by $68^{\circ} \mathrm{C}$ for $10 \mathrm{~min}$. DNA fragments obtained by $5^{\prime}$ and $3^{\prime}$ RACE PCR were subcloned into the pGEM-T vector and sequenced with ABI 3500 Genetic Analyzer (Applied Biosystems)

Reporter assays. Reporter assays employing the robol promoter luciferase reporters (Fig. 5a-h) were carried out as described previously ${ }^{13}$. The $p G L 3-p r o-$ moter vector carrying the 4.1, 2.7, 1.7, $0.9 \mathrm{~kb}$ (with or without 18 nucleotide deletion, $\triangle \mathrm{DR} 1$ ), or $0.5 \mathrm{~kb}$ robol promoter region ${ }^{13}$, the $p R L-T K$ Renilla luciferase vector (an internal control, Promega, E2241) and either the pact-FLAG-fruBM (a FruBM-expression vector) ${ }^{9}$, pact-FLAG-lola29M (a Lola29M-expression vector; present study), pact-FLAG-lola29M[K41R] (a Lola29M[K41R]-expression vector; present study), pact-FLAG-lola29M[ $\Delta 1$-300] (a Lola29M[ $\Delta 1-300]$-expression vector; present study), or pact-MCS (an empty vector) ${ }^{9}$ were co-transfected into S2 cell lines using FuGene HD Transfection Reagent (Promega, E2311). Forty-eight hours after transfection, the cells were lysed with a passive lysis buffer (Promega, E1910), and luciferase activity was measured using a Dual-Luciferase Reporter Assay System (Promega, E1910). To normalize the transfection efficiency, raw values for firefly luciferase activity were divided by raw Renilla luciferase activity values. The values representing fold suppression $(\mathrm{x})$ were calculated by dividing the normalized luciferase-activity values (lucA) in cells cotransfected with the empty vector pact$M C S$ by those (lucB) in cells cotransfected with pact-FLAG-fruBM (x=lucA/lucB). All experiments were carried out in triplicate, and data are presented as the fold suppression in mean $( \pm$ SEM) relative luciferase activity.
qPCR. qPCR (Fig. 4l) was performed using the LightCycler 1.0 system (Roche). Total RNA was extracted from the CNS of wandering third instar larvae using the RNeasy Mini Kit (Qiagen, 74104). To quantify robol expression levels, equal amounts of RNA were used to synthesize cDNA using the ReverTra Ace qPCR RT kit (TOYOBO, FSQ-101). Each cDNA was mixed with SYBR Premix Ex Taq II (TAKARA, RR820S) and 5 pmol of both forward (5'-CCACGCTCAACTGCAAAGTGGAG-3) and reverse (5'-AACTGGACGCGGTGCGATTTCTT-3) primers. RpL32 ( $r p 49)$ was amplified as an internal control using the primer pair: 5'-AGATCGTGAAGAAGCGCACCAAG-3' (forward) and 5'-

CACCAGGAACTTCTTGAATCCGG-3' (reverse). $\mathrm{qPCR}$ was conducted at $95^{\circ} \mathrm{C}$ for $30 \mathrm{sec}$ (initial denaturation), followed by 40 cycles of denaturation at $95^{\circ} \mathrm{C}$ for $5 \mathrm{~s}$, annealing at $55^{\circ} \mathrm{C}$ for $30 \mathrm{~s}$ and elongation at $72^{\circ} \mathrm{C}$ for $30 \mathrm{~s}$. Data processing was performed using LightCycler Software Ver. 3.5 (Roche).

Edman sequencing. To obtain a large amount of Lola29F sufficient for mass spectrometric analysis, we overexpressed the full-length lola29m tagged by V5 (UAS-lola29m-6xV5) under the control of fru-GAL4 in transgenic Drosophila. The brain-ventral nerve cord (CNS) complex was dissected from, in total, 1,000 larvae, and protein extracts from these CNSs were prepared using RIPA buffer $(50 \mathrm{mM}$ Tris-HCl, pH8.0, $150 \mathrm{mM} \mathrm{NaCl}, 0.5 \%$ Na-deoxycholate, $1.0 \%$ Triton X-100, $0.1 \%$ SDS, $1 \mathrm{mM}$ DTT, $1 \mu \mathrm{g} / \mathrm{ml}$ Leupeptin, $1 \mu \mathrm{g} / \mathrm{ml}$ Pepstatin, $0.5 \%$ Aprotinin), and subjected to immunoprecipitation as described above using $50 \mu$ Dynabeads protein $\mathrm{G}$ (Invitrogen, 10003D) and $5 \mu \mathrm{g}$ of the anti-V5 antibody (Invitrogen, 460705). The immunoprecipitate was then subjected to SDS-PAGE and transferred to PVDF membrane (APRO Science, BW-5201). After transfer, Lola29F visualized by coomassie staining (WAKO, 299-50101) was subjected to Edman degradation for protein sequencing. Protein sequencing was performed using Procise 494 HT Protein Sequencing System (Applied Biosystems, U.S.A.)

Electrophoretic Mobility Shift Assays (EMSA). EMSA (Fig. 5i-m) was carried out as described previously ${ }^{13}$. The $p M T$-3xflag-lola29m [K41R] and $p M T$-3xflaglola29m[ $\Delta 1-263]$ vectors were transfected together or individually into S2 cells, and the expression of proteins was induced by the addition of copper sulfate. The 3xFLAG-Lola29M[K41R] and 3xFLAG-Lola29M[ $\Delta 1-263]$ proteins were purified from S2 cell lysate using anti-FLAG resin columns in a FLAG HA Tandem Affinity Purification Kit (Sigma, TP0010) according to the manufacturer's protocol. EMSA experiments were performed using a DIG Gel Shift Kit (Roche, 03353591910). Binding reactions were established as follows: $1 \mathrm{x}$ binding buffer $(20 \mathrm{mM}$ Hepes, $\mathrm{pH}$ $7.5,10 \mathrm{mM}\left(\mathrm{NH}_{4}\right)_{2} \mathrm{SO}_{4}, 0.2 \%$ Tween $20,30 \mathrm{mM} \mathrm{KCl}$, and $\left.50 \mu \mathrm{M} \mathrm{ZnSO}{ }_{4}\right), 0.1 \mu \mathrm{g} / \mu \mathrm{l}$ poly[d(I-C)], $5 \mathrm{ng} / \mu \mathrm{l}$ poly-L-lysine, $0.8 \mathrm{ng}$ of a digoxigenin (DIG)-labeled probe containing the putative Lola29M-binding site, and $2 \mu \mathrm{l}$ of purified 3xFLAGLola29M[K41R] and/or 3xFLAG-Lola29M[ $\Delta 1-263]$ protein in a final volume of $10 \mu$ l. For competition reactions, a 25 -fold excess of unlabeled double-stranded oligonucleotide was included. Reactions were incubated at room temperature for $15 \mathrm{~min}$. For antibody-binding assays, $1 \mu \mathrm{g}$ of normal mouse IgG (Invitrogen, 10400C) or mouse anti-V5 (Invitrogen, 46-0705) was added to the binding reaction and the incubation proceeded for a further $15 \mathrm{~min}$. DIG-labeled DNA-protein complexes were detected by chemiluminescence using an ImageQuant LAS 4000 system (Fujifilm)

Mass spectrometric analysis. Aiming at identifying molecules that contribute to the N-terminal truncation of Lola29M, we carried out mass spectrometric analysis of proteins precipitated with a partially truncated Lola29M in the presence of the anti-V5 antibody. More specifically, the $p M T-H A-l o l a 29 m[\Delta 1-150]-V 5$ vector was transfected into $\$ 2$ cells, and the expression of proteins was induced by the addition of copper sulfate. The HA-Lola29M[ $\Delta 1-150]-\mathrm{V} 5$ products were immuno-purified using Anti-V5-tag mAb-Magnetic Beads (MBL, M167-11), trypsinized, and then directly subjected to LC-MS/MS analysis as previously described ${ }^{44}$. Briefly, extracted peptides were analyzed by ESI-MS/MS using an LTQ velos Orbitrap ETD instrument (Thermo Fisher Scientific, Pittsburgh, PA, USA). MS spectra were recorded over a range of $321-1600 \mathrm{~m} / \mathrm{z}$, followed by data-dependent collisioninduced dissociation (CID) MS/MS spectra generated from the 15 highest intensity precursor ions. For protein identification, spectra were processed using Proteome Discoverer 1.3.0.339 (Thermo Fisher Scientific) against MASCOT algorithm. For protein database searches, SwissProt (Taxonomy: Drosophila (fruit flies)) was used. The following parameters were used for the searches: tryptic cleavage, up to two missed cleavage sites, and tolerances of $\pm 10 \mathrm{ppm}$ for precursor ions, and $\pm 0.8 \mathrm{Da}$ for MS/MS fragment ions. MASCOT searches were performed allowing optional methionine oxidation and $\mathrm{N}$-terminal acetylation, and fixed cysteine carbamidomethylation. Peptide data were filtered using a Mascot significance threshold of 0.05 as valid identification

Detection of ubiquitinated Lola29M. To detect ubiquitination of Lola29M (Supplementary Fig. 8), Lola29M-3xFLAG was overexpressed in S2 cells. $1 \mu \mathrm{g}$ of the pMT-lola29m-3xFLAG vector was transfected into $\mathrm{S} 2$ cells $\left(1 \times 10^{7}\right.$ cells $)$ with or without $1 \mu \mathrm{g}$ or $3 \mu \mathrm{g}$ of the $p M T-f r u b m$ vector, and the expression of proteins was induced by the addition of copper sulfate. The Lola29M-3xFLAG protein was purified from S2 cell lysate using anti-FLAG resin columns in a FLAG HA Tandem Affinity Purification Kit (Sigma, TP0010). The immunoprecipitate was then 
analyzed by western blotting with a primary antibody, mouse anti-Ub-K48 (1:500, abcam ab140601) and a secondary antibody, HRP-conjugated anti-mouse IgG (1:3000, Sigma). Fluorescent images were obtained using ImageQuant LAS 4000 (Fujifilm).

Statistical analysis. Statistical analyses were done by GraphPad Prism 7.0b software.

Reporting Summary. Further information on experimental design is available in the Nature Research Reporting Summary linked to this article.

\section{Data availability}

The data sets generated for this manuscript are available from the corresponding author upon reasonable request. List of proteins that were immuno-purified with Lola29M protein is found in Supplementary Table 1. Primer sequence data sets are found in Supplementary Tables 2, 3. DNA probes used in EMSA experiments are included in Supplementary Table 4. The source data underlying Figs. 1, 2, 5 and 6 and Supplementary Figs 3, 5, 6, 7, 8, 13, 14 are provided as a Source Data file.

Received: 4 June 2018 Accepted: 11 December 2018.

Published online: 11 January 2019

\section{References}

1. Yang, C. F. \& Shah, N. M. Representing sex in the brain, one module at a time. Neuron 82, 261-278 (2014).

2. Cachero, S., Ostrovsky, A. D., Yu, J. Y., Dickson, B. J. \& Jefferis, G. S. X. E. Sexual dimorphism in the fly brain. Curr. Biol. 20, 1589-1601 (2010).

3. Costa, M., Manton, J. D., Ostrovsky, A. D., Prohaska, S. \& Jefferis, G. S. X. E. NBLAST: Rapid, sensitive comparison of neuronal structure and construction of neuron family databases. Neuron 91, 293-311 (2016).

4. Kimura, K.-i, Ote, M., Tazawa, T. \& Yamamoto, D. Fruitless specifies sexually dimorphic neural circuitry in the Drosophila brain. Nature 438, 229-233 (2005).

5. Kimura, K.-i, Hachiya, T., Koganezawa, M., Tazawa, T. \& Yamamoto, D. Fruitless and Doublesex coordinate to generate male-specific neurons that can initiate courtship. Neuron 59, 759-769 (2008).

6. Yu, J. Y., Kanai, M. I., Demir, E., Jefferis, G. S. X. E. \& Dickson, B. J. Cellular organization of the neural circuit that drives Drosophila courtship behavior. Curr. Biol. 20, 1602-1614 (2010).

7. Zollman, S., Godt, D., Prive, G. G., Couderc, J. L. \& Laski, F. A. The BTB domain, found primarily in zinc finger proteins, defines an evolutionarily conserved family that includes several developmentally regulated genes in Drosophila. Proc. Natl Acad. Sci. USA 91, 10717-10721 (1994).

8. Zhang, W. et al. A developmentally regulated splice variant from the complex lola locus encoding multiple different zinc finger domain proteins interacts with the chromosomal kinase JIL-1. J. Biol. Chem. 278, 11696-11704 (2003).

9. Ito, H. et al. Fruitless recruits two antagonistic chromatin factors to establish single-neuron sexual dimorphism. Cell 149, 1327-1338 (2012).

10. Dickson, B. J. Wired for sex: the neurobiology of Drosophila mating decisions. Science 322, 904-909 (2008).

11. Pavlou, H. J. \& Goodwin, S. F. Courtship behavior in Drosophila melanogaster: towards a 'courtship connectome'. Curr. Opin. Neurobiol. 23, 76-83 (2013).

12. Yamamoto, D. \& Koganezawa, M. Genes and circuits of courtship behaviour in Drosophila males. Nat. Rev. Neurosci. 14, 681-692 (2013).

13. Ito, H., Sato, K., Kondo, S., Ueda, R. \& Yamamoto, D. Fruitless repressesrobo1 transcription to shape male-specific neural morphology and behavior in Drosophila. Curr. Biol. 26, 1532-1542 (2016).

14. Gilles, G. E., Pienaar, I. S., Vohra, S. \& Qamhawi, Z. Sex differences in Parkinson's disease. Front. Neuroendocrinol. 35, 370-398 (2014).

15. Toba, G. et al. The gene search system. A method for efficient detection and rapid molecular identification of genes in Drosophila melanogaster. Genetics 151, 725-737 (1999).

16. Goto, J., Mikawa, Y., Koganezawa, M., Ito, H. \& Yamamoto, D. Sexually dimorphic shaping of interneuron dendrites involves the Hunchback transcription factor. J. Neurosci. 31, 5454-5459 (2011).

17. Giniger, E., Tieje, K., Jan, L. Y. \& Jan, Y. N. lola encodes a putative transcription factor required for axon growth and guidance in Drosophila. Development 120, 1385-1398 (1994).

18. Crowner, D., Madden, K., Goeke, S. \& Giniger, E. Lola regulates midline crossing of CNS axons in Drosophila. Development 129, 1317-1325 (2002).

19. Davies, E. L. et al. The transcriptional regulator lola is required for stem cell maintenance and germ cell differentiation in the Drosophila testis. Dev. Biol. 373, 310-321 (2013).
20. Dinges, N., Morin, V., Kreim, N., Southall, T. D. \& Roignant, J. Y. Comprehensive characterization of the complex lola locus reveals a novel role in the octopaminergic pathway via tyramine $\beta$-hydroxylase regulation. Cell Rep. 21, 2911-2925 (2017).

21. Clowney, E. J., Iguchi, S., Bussell, J. J., Scheer, E. \& Ruta, V. Multimodal chemosensory circuits controlling male courtship in Drosophila. Neuron 87, 1036-1049 (2015).

22. Kallman, B. R. \& Scott, K. Excitation and inhibition onto central courtship neurons biases Drosophila mate choice. eLife 4, e11188 (2015).

23. Koganezawa, M., Haba, D., Matsuo, T. \& Yamamoto, D. The shaping of male courtship posture by lateralized gustatory inputs to male-specific interneurons. Curr. Biol. 20, 1-8 (2010).

24. Herhaus, L. \& Dikic, I. Expanding the ubiquitin code through posttranslational modification. EMBO Rep. 16, 1071-1083 (2015).

25. Possidente, D. R. \& Murphey, R. K. Genetic control of sexually dimorphic axon morphology in Drosophila sensory neurons. Dev. Biol. 132, 448-457 (1989).

26. Mathew, R. et al. BTB-ZF factors recruit the E3 ligase cullin 3 to regulate lymphoid effector programs. Nature 491, 618-621 (2012).

27. Collins, G. A. \& Goldberg, A. L. The logic of the $26 \mathrm{~S}$ proteasome. Cell 169, 792-806 (2017)

28. Dhordain, P. et al. The BTB/POZ domain targets the LAZ3/BCL6 oncoprotein to nuclear dots and mediates homomerisation in vivo. Oncogene 11, 2689-2697 (1995)

29. Neville, M. C. et al. Male-specific Fruitless isoforms target neurodevelopmental genes to specify a sexually dimorphic nervous system. Curr. Biol. 24, 229-241 (2014).

30. Kidd, T. et al. Roundabout controls axon crossing of the CNS midline and defines a novel subfamily of evolutionarily conserved guidance receptors. Cell 92, 205-215 (1998).

31. Aza-Blanc, P., Ramirez-Weber, F. A., Laget, M. P., Schwarz, C. \& Kornberg, T. B. Proteolysis that is inhibited by hedgehog targets Cubitus interruptus protein to the nucleus and converts it to a repressor. Cell 89, 1043-1053 (1997).

32. Chen, X., Rahman, R., Guo, F. \& Rosbash, M. Genome-wide identification of neuronal activity-regulated genes in Drosophila. eLife 5, e19942 (2016).

33. Halaska, J. M., Lee, K. K., Kowalski, A. K. \& Wilson, K. L. Transcriptional repressor germ-cell-less (GCL) and barrier to autointegration factor (BAF) compete for binding to emerin in vitro. J. Biol. Chem. 278, 6969-6975 (2003).

34. Nili, E. et al. Nuclear membrane protein LAP2 $\beta$ mediates transcriptional repression alone and together with its binding partner GCL (germ-cell-less). J. Cell. Biol. 114, 3297-3307 (2001).

35. Pae, J., Cinalli, R. M., Marzio, A., Pagano, M. \& Lehmann, R. GCL and CUL3 control the switch between cell lineages by mediating localized degradation of an RTK. Dev. Cell 42, 130-142 (2017).

36. Werner, A. et al. Cell-fate determination by ubiquitin-dependent regulation of translation. Nature 525, 523-527 (2015).

37. Jenett, A. et al. A GAL4-driver line resource for Drosophila neurobiology. Cell Rep. 2, 991-1001 (2012)

38. Usui-Aoki, K. et al. Formation of the male-specific muscle in female Drosophila by ectopic fruitless expression. Nat. Cell Biol. 8, 500-506 (2000).

39. Song, H. J. et al. The fruitless gene is required for the proper formation of axonal tracts in the embryonic central nervous system of Drosophila. Genetics 162, 1703-1724 (2002).

40. Pfeiffer, B. D., Truman, J. W. \& Rubin, G. M. Using translational enhancers to increase transgene expression in Drosophila. Proc. Natl Acad. Sci. USA 109, 6626-6631 (2012)

41. Hayashi, S. Male-specific GFP marker strain of Drosophila melanogaster. Dros. Inf. Serv. 93, 224-225 (2010).

42. Lee, T. \& Luo, L. Mosaic Analysis with a repressible cell marker for studies of gene function in neuronal morphogenesis. Neuron 22, 451-461 (1999).

43. Zhang, Z. \& Yuan, K.-H. Practical Statistical Power Analysis Using Webpower and $R$ (pp. 350. ISDSA Press, Notre Dame, IN, 2018. https://lccn.loc.gov/ 2018901582 LCCN Permalink.

44. Yokoyama, A. et al. Identification of Myelin Transcription Factor 1 (MyT1) as a subunit of the neural cell type-specific Lysine-specific Demethylase 1 (LSD1) complex. J. Biol. Chem. 289, 18152-18162 (2014).

\section{Acknowledgements}

We thank the Bloomington Drosophila Stock Center, Drosophila Genetic Resource Center at Kyoto, and Vienna Drosophila Stock Center for fly stocks, and Azusa Utsum for secretarial assistance. This work was supported, in part, by Grants-in-Aid for Scientific Research from MEXT to D.Y. (Nos. 17K19371, 17H05935 and 16H06371), to K.S. (Nos. 17K07040, 25132702 and 24700309), to A.Y. (No. 16K08606) and to H.I. (No. 16K06985) and a Life Science Grant from the Takeda Science Foundation (D.Y. and K.S.). 


\section{Author contributions}

K.S. and D.Y. planned the project, K.S. designed and performed the experiments, A.Y. performed mass spectrometric analyses, K.S., G.T. and H.I. analyzed the data, and K.S. and D.Y. wrote the paper.

\section{Additional information}

Supplementary Information accompanies this paper at https://doi.org/10.1038/s41467018-08146-1.

Competing interests: The authors declare no competing interests.

Reprints and permission information is available online at http://npg.nature.com/ reprintsandpermissions/

Journal peer review information: Nature Communications thanks the anonymous reviewers for their contribution to the peer review of this work. Peer reviewer reports are available.
Publisher's note: Springer Nature remains neutral with regard to jurisdictional claims in published maps and institutional affiliations.

(c) (i) Open Access This article is licensed under a Creative Commons Attribution 4.0 International License, which permits use, sharing, adaptation, distribution and reproduction in any medium or format, as long as you give appropriate credit to the original author(s) and the source, provide a link to the Creative Commons license, and indicate if changes were made. The images or other third party material in this article are included in the article's Creative Commons license, unles indicated otherwise in a credit line to the material. If material is not included in the article's Creative Commons license and your intended use is not permitted by statutory regulation or exceeds the permitted use, you will need to obtain permission directly from the copyright holder. To view a copy of this license, visit http://creativecommons.org/ licenses/by/4.0/.

(C) The Author(s) 2019 